\title{
Molecular characterization and pathogenicity of fungal taxa associated with cherry leaf spot disease
}

\section{Chethana KWT ${ }^{1,2}$, Jayawardene RS $^{2}$, Zhang $\mathbf{W}^{1}$, Zhou YY ${ }^{1}$, Liu $\mathbf{M}^{1}$, Hyde KD ${ }^{2}$, Li XH ${ }^{1}$, Wang $\mathrm{J}^{3}$, Zhang $\mathrm{KC}^{3}$ and Yan JY ${ }^{1}$}

\author{
${ }^{1}$ Beijing Key Laboratory of Environment Friendly Management on Fruit Diseases and Pests in North China, Institute of \\ Plant and Environment Protection, Beijing Academy of agriculture and forestry sciences, Beijing, China \\ ${ }^{2}$ Center of Excellence in Fungal Research, Mae Fah Luang University, Chiang Rai, Thailand \\ ${ }^{3}$ Academy of Forestry and Pomology Sciences, Beijing, China
}

Chethana KWT, Jayawardene RS, Zhang W, Zhou YY, Liu M, Hyde KD, Li XH, Wang J, Zhang KC, Yan JY 2019 - Molecular characterization and pathogenicity of fungal taxa associated with cherry leaf spot disease. Mycosphere 10(1), 490-530, Doi 10.5943/mycosphere/10/1/8

\begin{abstract}
Cherry leaf spot is one of the most common and devastating diseases of cherries worldwide. The disease causes considerable yield losses in many cherry growing regions. We surveyed cherry leaf spot disease in Beijing City and collected 67 fungal isolates from approximately 60 diseased leaves. Multigene phylogenetic analyses coupled with morphological observations facilitated the identification of species isolated from the diseased tissues. Pathogenicity assays were conducted for six isolates representing all the identified species and Koch's postulates were confirmed on three cultivars of Prunus avium under greenhouse conditions. These results confirmed their pathogenicity on cherry leaves as symptoms were reproduced. Based on these results, a novel taxon Alternaria prunicola sp. nov. is reported as the main pathogen of Cherry leaf spot in Beijing City. In addition, Alternaria alternata, Alternaria pseudoeichhorniae sp. nov., Colletotrichum aenigma, Colletotrichum pseudotheobromicola sp. nov., Epicoccum pseudokeratinophilum sp. nov., Nothophoma pruni sp. nov., Nothophoma quercina and Stagonosporopsis citrulli were also isolated from Cherry leaf spots. Significant variations in the virulence were observed among fungal species on different cherry cultivars.
\end{abstract}

Key words - Alternaria - Colletotrichum - disease management - Epicoccum - Nothophoma pathogenicity - Prunus - Stagonosporopsis - 5 new species

\section{Introduction}

Cherry (Prunus avium L., Rosaceae) is an important stone fruit of temperate regions with many beneficial properties. Different cultivars of cherries including sweet cherry, sour cherry, flowering ornamental cherries and wild cherries are cultivated worldwide for their economic value (Holb 2009, Faust \& Surányi 2011, Joshua 2012, USDA 2017). According to the statistical data from FAO (2018), world cherry cultivation and production have reached 0.44 million hectares (Mha) and 2.3 million tonnes in 2016. Due to extreme weather conditions, world cherry production forecasted to drop by $3 \%$ by 2018 (USDA 2017). Driven by ongoing consumer demand and high market value, cherry cultivation has expanded in China from top cherry producing provinces such as Shandong and Liaoning to inland provinces including Gansu, Henan, Shaanxi, Shanxi and 
Yunnan (Scott et al. 2014). In the year 2016, cherry cultivation in China has increased to 9.034 kha resulting an increased production of 0.037 Million tonnes (FAO 2018). Currently, many cherry cultivars are grown in China, most of which are introduced from other countries. Among them 'Brooks' or 'Red Lantern' is known as the most dominant cultivar (Scott et al. 2014). Even with ideal conditions, cherries are susceptible to many diseases including bacterial, fungal, parasitic and viral diseases (Uyemoto et al. 2018). Among these, Cherry leaf spot disease is one of the most common and widely spread diseases in many cherry growing regions in the world with humid conditions (Garcia \& Jones 1993, Ellis 2008, Farr \& Rossman 2011, Choi et al. 2014) and affect leaves, leaf stems, fruits, and fruit stem causing defoliation and eventual death of the tree (Jones \& Sutton 1996, Díaz et al. 2007, Farr \& Rossman 2011).

Cherry leaf spot disease was first observed in the USA and then reported from other countries, mainly in mainland Europe, Turkey, China, Chile, Japan and Russia (Ogawa \& English 1991, Holb 2009). Several names including cherry leaf blight, cherry anthracnose (Holb 2009) and yellow leaf and shot hole disease have been used in literature to address the same disease (Ogawa \& English 1991). Blumeriella jaapii (Rehm) Arx. (asexual morph: Phloeosporella padi (Lib.) Arx.; Cylindrosporium padi $\mathrm{P}$. Karst.) was identified as the main pathogen of Cherry leaf spot disease in many countries (Sjulin et al. 1989, Heald 1993, Joshua \& Mmbaga 2013). Over the years, this pathogen has been synonymized under different names (Index Fungorum 2018). In addition to Blumeriella jaapii, several other pathogens have been also identified as causative agents of Cherry leaf spot disease. Pseudocercospora pruni-persicicola (J.M. Yen) J.M. Yen has been isolated from cherry leaf spots in Taiwan and Korea (Farr \& Rossman 2013, Choi et al. 2014). In addition, Alternaria cerasi Potebnia. and Alternaria alternata (Fr.) Keissl. have been reported from cherry leaf spots in China and in Greece respectively (Zhu \& Chang 2004, Thomidis \& Tsipouridis 2006). Most of the studies conducted on the disease have focused on B. jaapii infection (Schuster \& Tobutt 2004, Díaz et al. 2007, Joshua \& Mmbaga 2013). Previous studies have characterized the cherry leaf spot symptoms common to all the pathogens, which initiate by forming pinpoint lesions on the leaf surface. These rapidly enlarging lesions change their colour to brown and coalesced together to form large patches of necrotic tissues (Ellis 2008, Holb 2009, Khan et al. 2014). According to Holb (2009) and Higgins (1914), an absiss layer forms around the leaf spot causing rapid cell enlargement which results in the formation of a shot hole. Shot holes with round and evenly curved smooth margins are characteristic for cherry leaf spot disease (Higgins 1914). Heavily infected leaves fall to the ground leading to premature defoliation, a major symptom of cherry leaf spot, and facilitate the fungus to overwinter among the leaf debris by switching its lifestyle (Ellis 2008, Holb 2009). In response to warm and humid weather conditions, conidia discharge and establish new infections (Eisensmith \& Jones 1981, Holb 2009). Therefore, timely execution of control strategies can be effective against these pathogens.

There are few reports on Cherry leaf spot disease in China from provinces including Henan and Shandong (Zhu \& Chang 2004, Shu-gui 2007, Li et al. 2011, Zhao \& Liu 2012). Alternaria alternata (Zhao \& Liu 2012) and Alternaria cerasi Potebnia. (Zhu \& Chang 2004) have been reported as the causative agents of cherry leaf spots in China. However, detailed morphological studies combined with phylogenetic analyses based on DNA sequence data and pathogenicity assays have not been conducted. We have observed severe leaf spot conditions in cherry orchards in Beijing during 2016 and 2018. Surprisingly, no studies have been conducted in Beijing yet to identify the causal agent of the disease. Therefore, in the current study, we studied phytopathogenic fungal species associated with cherry leaf spot symptoms in Beijing, China; identified them based on morphology and DNA sequence data, investigate their phylogeny and assess their pathogenicity. Our main objective is to generate information needed to guide species-specific disease management of cherry leaf spot disease and to evaluate the source of the primary inoculum.

\section{Materials \& Methods}

\section{Sample collection, fungal isolation, and morphological characterization}


Cherry leaves with leaf spot symptoms were collected from orchards at Beijing Academy of Forestry and Pomology Sciences, Beijing, China during the springs of 2016 and 2018. Symptomatic leaves were brought to the laboratory in separate plastic bags. Samples were surface sterilized by washing with $1 \%$ sodium hypochlorite for $1 \mathrm{~min}, 70 \%$ ethanol for $1 \mathrm{~min}$, rinsed three times in sterilized water, blotted dry and placed on Potato Dextrose Agar (PDA) and on Potato Carrot Agar (PCA). Cultures were maintained at $25{ }^{\circ} \mathrm{C}$ for $2-5$ days. Fungal structures were observed and photographed using an Axio Imager Z2 photographic microscope (Carl Zeiss Microscopy, Oberkochen, Germany) and measurements were taken using ZEN PRO 2012 software (Carl Zeiss Microscopy, Germany). Fourty conidial measurements were made per isolate. Growth rates and cultural characteristics were recorded after 5 days. The growth rate was calculated as the mean of two perpendicular measurements. Herbarium specimens were deposited in the Mae Fah Luang University (MFLU) herbarium, Thailand, and the Herbarium of Cryptograms (HKAS), Kunming Institute of Botany, China. Cultures were deposited in the culture collections at Mae Fah Luang University, Thailand (MFLUCC), the culture collection at Kunming Institute of Botany (KUMCC), China, and the Beijing Academy of Agricultural and Forestry Sciences (JZB) culture collection, China. Facesoffungi and MycoBank numbers were acquired as in Jayasiri et al. (2015) and (Myco Bank 2018).

\section{DNA extraction, PCR amplification and sequencing}

Genomic DNA was extracted from 67 representative isolates using the modified protocol described in Chethana et al. (2017). The following loci were amplified using the primers given in Table 1. For Alternaria species: ITS, GAPDH ${ }_{\mathrm{ALT}}$, RPB2, TEF 1- $\alpha$ and Alt-a 1; For Colletotrichum species: ITS, GAPDH, , CHS-1, ACT and TUB2; For Didymellaceae species: LSU, ITS, RPB2 and TUB2. Polymerase chain reactions (PCR) were conducted in an Applied Biosystems Vetri Thermal Cycler with the following PCR conditions for ITS, RPB2, and TEF 1- $\alpha$ regions (White et al. 1990): initial denaturation for $3 \mathrm{~min}$ at $95{ }^{\circ} \mathrm{C}$, followed by 34 cycles of denaturation for $30 \mathrm{~s}$ at $95{ }^{\circ} \mathrm{C}$ and $30 \mathrm{~s}$ of annealing and $1 \mathrm{~min}$ elongation at $72{ }^{\circ} \mathrm{C}$, and a final extension for $10 \mathrm{~min}$ at $72{ }^{\circ} \mathrm{C}$. The annealing temperatures were as follows: $52{ }^{\circ} \mathrm{C}$ for LSU, $54{ }^{\circ} \mathrm{C}$ for $\mathrm{GADPH}_{\mathrm{C}}$ and TEF $1-\alpha, 56{ }^{\circ} \mathrm{C}$ for ACT, RPB2 and TUB2, and $59{ }^{\circ} \mathrm{C}$ for CHS-1 and ITS gene regions. Slightly deviated PCR conditions were provided for the other two genes: initial denaturation for $2 \mathrm{~min}$ at $94{ }^{\circ} \mathrm{C}$, followed by 35 cycles of denaturation for $1 \mathrm{~min}$ at $96{ }^{\circ} \mathrm{C}, 1 \mathrm{~min}$ of annealing at $58{ }^{\circ} \mathrm{C}$ and $1 \mathrm{~min}$ elongation at $72{ }^{\circ} \mathrm{C}$ and a final extension for $10 \mathrm{~min}$ at $72{ }^{\circ} \mathrm{C}$ for the $\mathrm{GAPDH}_{\mathrm{ALT}}$; initial denaturation for $2 \mathrm{~min}$ at $94{ }^{\circ} \mathrm{C}$, followed by 35 cycles of denaturation for $1 \mathrm{~min}$ at $94{ }^{\circ} \mathrm{C}, 30 \mathrm{~s}$ of annealing at $57{ }^{\circ} \mathrm{C}$ and $45 \mathrm{~s}$ elongation at $72{ }^{\circ} \mathrm{C}$, and a final extension for $10 \mathrm{~min}$ at $72{ }^{\circ} \mathrm{C}$ for Alt-a 1 . The PCR solution mixture was composed of $0.3 \mathrm{ml}$ of TaKaRa Ex-Taq DNA polymerase (TaKaRa, China), $2.5 \mathrm{ml}$ of 10x Ex-Taq buffer (TaKaRa, China), $3.0 \mathrm{ml}$ of dNTPs (TaKaRa, China), $1 \mathrm{ml}$ of genomic DNA, 1 $\mathrm{ml}$ of each primer, and $16.2 \mathrm{ml}$ of ddH2O. The PCR products were visualized on $1 \%$ agarose gel under UV light using a GelDoc XR+Molecular Imager (BIO-RAD, USA) after ethidium bromide staining. Sequencing of PCR products was carried at the Sunbiotech Company, Beijing, China. The sequences from forward and reverse primers were used to obtain consensus sequences with DNAMAN 6.0 (Lynnon Biosoft, USA). Those sequences were deposited in the GenBank and their accession numbers are given in Supplementary Tables 2-4.

\section{Phylogenetic analysis}

Based on the analyses conducted using the National Center for Biotechnology Information (NCBI) search engine GenBank BLASTn, fungal members similar to the new taxa were included in the analyses. Reference sequences were obtained from GenBank (Weir et al. 2012, Woudenberg et al. 2013, 2015, Chen et al. 2015, 2017, Abdel-Wahab et al. 2017, Crous et al. 2017, Jayasiri et al. 2017, Thambugala et al. 2017, Hyde et al. 2017, 2018, Tibpromma et al. 2018, Valenzuela-Lopez et al. 2018, Wanasinghe et al. 2018) are listed in Tables 2-4. Individual datasets for the genes were aligned using the default settings of MAFFT 7 (Katoh \& Toh 2008, http://mafft.cbrc.jp/alignment/server) and improved manually where necessary using BIOEDIT 
(Hall 1999). Aligned gene regions were concatenated for the analyses in the following order: ITS, GAPDH $_{\mathrm{ALT}}$, RPB2, TEF 1- $\alpha$ and Alt-a 1 for Alternaria species; ITS, GAPDH $\mathrm{C}, \mathrm{CHS}-1$, ACT and TUB2 for Colletotrichum species; LSU, ITS, RPB2, and TUB2 for Didymellaceae species.

Maximum parsimony (MP) analysis based on the combined dataset was conducted in PAUP (phylogenetic analysis using parsimony) 4.0b10 (Swofford 2002). Phylogenetic trees were generated using the heuristic search option with tree bisection-reconnection (TBR) branch swapping and 1000 random sequence additions. Ambiguous regions in the alignment were excluded, and gaps were treated as missing data. Clade stability was assessed using a bootstrap analysis with 1000 replications (Hillis \& Bull 1993). Maxtrees were set up to 1000, branches of zero length were collapsed, and all multiple parsimonious trees were saved. Descriptive statistics such as tree length (TL), consistency index (CI), retention index (RI), relative consistency index (RC), and homoplasy index (HI) were calculated for trees inferred under different optimality criteria. Their significance was evaluated using Kishino-Hasegawa tests (KHT) (Kishino \& Hasegawa 1989).

Maximum likelihood (ML) analysis was conducted in RAXMLGUI 0.9b2 (Silvestro \& Michalak 2010) for 1000 nonparametric bootstrapping iterations, using the general time reversible model (GTR) with a discrete gamma distribution. Trees with the final likelihood values of 6586.660252, -8409.454513, -16 769.656375 were selected as the best-scoring trees for Alternaria species, Colletotrichum species and Didymellaceae species respectively and the replicates were plotted relative to it.

Furthermore, Bayesian reconstructions were performed using MRBAYES 3.0b4 (Ronquist \& Huelsenbeck 2003). The evolutionary models for phylogenetic analyses were determined independently for each locus by MRMODELTEST 2.3 (Nylander 2004). The best model selected for each locus was given in Table 1. Four simultaneous Markov chains were run for 1,000,000 generations with increments of additional generations when needed until the standard deviation of split frequencies reach 0.01 . From the 10000 trees obtained, first 2000 representing the burn-in phase of the analyses were discarded while the remaining 8,000 trees were used for calculating posterior probabilities in the majority rule consensus tree (critical value for the topological convergence diagnostic set to 0.01) (Crous et al. 2006). All the phylogenetic trees were drawn using TREEVIEW 1.6.6 (Page 1996). Sequences derived in this study were deposited in GenBank; alignments and trees were deposited in TreeBase for Alternaria species, Colletotrichum species and Didymellaceae species (S23535).

\section{Pathogenicity assay}

Pathogenicity tests were conducted on young, healthy detached leaves of three Prunus avium cultivars namely, 'Tieton', 'Summit' and 'Sunburst' from Tongzhou Experimental Station for Cherries, Beijing Academy of Forestry and Pomology Sciences, Beijing, China. Pathogenicity of three isolates of Alternaria species (MFLUCC 18-1597, MFLUCC 18-1599, and MFLUCC 181587), three Didymellaceae species (MFLUCC 18-1593, MFLUCC 18-1595 and MFLUCC 181600) and three isolates Colletotrichum species (MFLUCC 18-1604, MFLUCC 18-1603 and MFLUCC 18-1602) were randomly tested. Leaf surfaces were sterilized by washing in $75 \%$ ethanol for $1 \mathrm{~min}$, then in $10 \%$ sodium hypochlorite for $1 \mathrm{~min}$, followed by washing with distilled water three times. The experiment was conducted using six leaves per isolate (including one control), inoculated by non-wound and wound inoculation approaches and repeated three times to get stable results. These wound and non-wound inoculations were performed on symmetrical halves of each leaf. For the wound inoculation, the upper epidermal layer of the leaf was injured

with a sterile needle. One hundred $\mu 1$ of the inoculum consisting of $10^{6}$ spores $/ \mathrm{ml}$ conidial suspension were inoculated on both the wound and non-wound sites of each of the leaves. Sterile water was used as the control. Each inoculated leaf was placed in a $12 \mathrm{~cm}$ diameter petri dish and incubated in a moist chamber at $25{ }^{\circ} \mathrm{C}$ with an $80 \%$ relative humidity until symptoms appeared. Lesion lengths were recorded three days after the inoculation. Koch's postulates were confirmed by 
re-isolating the inoculated fungus. The re-isolated fungus was identified based on cultural and morphological characters.

Table 1 Primers used in the study, with sequences, their sources, sizes and evolutionary models used in the study

\begin{tabular}{|c|c|c|c|c|c|c|}
\hline Gene & Product name & Primer & Sequence (5'-3') & Product size & $\begin{array}{l}\text { Nucleotide } \\
\text { substitution } \\
\text { model }\end{array}$ & Reference \\
\hline ACT & Actin & $\begin{array}{l}\text { ACT-512F } \\
\text { ACT-783R }\end{array}$ & $\begin{array}{l}\text { ATG TGC AAG GCC GGT TTC GC } \\
\text { TAC GAG TCC TTC TGG CCC AT }\end{array}$ & $256 \mathrm{bp}$ & $\mathrm{HKY}+\mathrm{G}$ & $\begin{array}{l}\text { Carbone \& } \\
\text { Kohn (1999) }\end{array}$ \\
\hline Alt-a 1 & $\begin{array}{l}\text { Alternaria major allergen } \\
1\end{array}$ & $\begin{array}{l}\text { Alt-F } \\
\text { Alt-R }\end{array}$ & $\begin{array}{l}\text { ATG CAG TTC ACC ACC ATC GC } \\
\text { ACG AGG GTG AYG TAG GCG TC }\end{array}$ & $457 \mathrm{bp}$ & $\mathrm{TrNef}+\mathrm{G}$ & $\begin{array}{l}\text { Hong et al. } \\
(2005)\end{array}$ \\
\hline CHS-1 & Chitin synthase & $\begin{array}{l}\text { CHS-79F } \\
\text { CHS-345R }\end{array}$ & $\begin{array}{l}\text { TGG GGC AAG GAT GCT TGG AAG AAG } \\
\text { TGG AAG AAC CAT CTG TGA GAG TTG }\end{array}$ & $282 \mathrm{bp}$ & $\mathrm{TrNef}+\mathrm{G}$ & $\begin{array}{l}\text { Carbone \& } \\
\text { Kohn (1999) }\end{array}$ \\
\hline $\begin{array}{l}\text { GAPD } \\
\mathrm{H}_{\mathrm{ALT}}\end{array}$ & $\begin{array}{l}\text { glyceraldehyde-3- } \\
\text { phosphate dehydrogenase }\end{array}$ & $\begin{array}{l}\text { gpd1 } \\
\text { gpd2 }\end{array}$ & $\begin{array}{l}\text { CAA CGG CTT CGG TCG CAT TG } \\
\text { GCC AAG CAG TTG GTT GTG C }\end{array}$ & $535 \mathrm{bp}$ & $\mathrm{TrN}+\mathrm{G}$ & $\begin{array}{l}\text { Berbee et al. } \\
\text { (1999) }\end{array}$ \\
\hline $\begin{array}{l}\text { GAPD } \\
\mathrm{H}_{\mathrm{C}}\end{array}$ & $\begin{array}{l}\text { glyceraldehyde-3- } \\
\text { phosphate dehydrogenase }\end{array}$ & $\begin{array}{l}\text { GDF } \\
\text { GDR }\end{array}$ & $\begin{array}{l}\text { GCC GTC AAC GAC CCC TTC ATT GA } \\
\text { GGG TGG AGT CGT ACT TGA GCA TGT }\end{array}$ & $250 \mathrm{bp}$ & $\mathrm{HKY}+\mathrm{G}$ & $\begin{array}{l}\text { Templeton et } \\
\text { al. (1992) }\end{array}$ \\
\hline ITS & $\begin{array}{l}\text { Internal transcribed } \\
\text { spacer }\end{array}$ & $\begin{array}{l}\text { ITS } 1 \\
\text { ITS } 4\end{array}$ & $\begin{array}{l}\text { TCC GTA GGT GAA CCT GCG G } \\
\text { TCC TCC GCT TAT TGA TAT GC }\end{array}$ & $\begin{array}{l}\text { Alternaria: } 539 \\
\mathrm{bp} \\
\text { Colletotrichum } \\
: 533 \mathrm{bp} \\
\text { Didymellaceae } \\
: 486 \mathrm{bp}\end{array}$ & $\begin{array}{l}\text { Alternaria: } \\
\text { SYM+I+G } \\
\text { Colletotrichum } \\
: \text { TrNef+G } \\
\text { Didymellaceae } \\
\text { : TrNef }+\mathrm{I}+\mathrm{G}\end{array}$ & $\begin{array}{l}\text { White et al. } \\
\text { (1990) }\end{array}$ \\
\hline LSU & $\begin{array}{l}\text { 28S large subunit of } \\
\text { nuclear ribosomal RNA }\end{array}$ & LROR & ACC CGC TGA ACT TAA GC & $877 \mathrm{bp}$ & TrNef+I & $\begin{array}{l}\text { Vilgalys \& } \\
\text { Hester } \\
(1990) \\
\text { Rehner \& } \\
\text { Samuels } \\
(1994)\end{array}$ \\
\hline $\mathrm{RPB}_{2}$ & $\begin{array}{l}\text { RNA polymerase II } \\
\text { second largest subunit }\end{array}$ & $\begin{array}{l}\text { RPB2-5F } \\
\text { RPB2-7cR }\end{array}$ & $\begin{array}{l}\text { GGG GWG AYC AGA AGA AGG C } \\
\text { CCC ATR GCT TGY TTR CCC AT }\end{array}$ & $\begin{array}{l}\text { Alternaria: } 881 \\
\text { bp } \\
\text { Didymellaceae } \\
: 905 \mathrm{bp}\end{array}$ & $\begin{array}{l}\text { Alternaria: } \\
\text { TrNef+G } \\
\text { Didymellaceae } \\
\text { : TIM2ef }+\mathrm{I}+\mathrm{G}\end{array}$ & $\begin{array}{l}\text { Liu et al. } \\
(1999) \\
\text { Sung et al. } \\
(2007)\end{array}$ \\
\hline
\end{tabular}


Table 1 Continued

\begin{tabular}{|c|c|c|c|c|c|c|}
\hline Gene & Product name & Primer & Sequence $\left(5^{\prime}-3^{\prime}\right)$ & Product size & $\begin{array}{l}\text { Nucleotide } \\
\text { substitution } \\
\text { model }\end{array}$ & Reference \\
\hline TEF $1-\alpha$ & $\begin{array}{l}\text { Partial translation } \\
\text { elongation } \\
\text { factor } 1-\alpha\end{array}$ & $\begin{array}{l}\text { TEF1-728F } \\
\text { TEF1- } \\
\text { 986R }\end{array}$ & $\begin{array}{l}\text { CAT CGA GAA GTT CGA GAA GG } \\
\text { TAC TTG AAG GAA CCC TTA CC }\end{array}$ & $\begin{array}{l}\text { Alternaria: } 223 \\
\text { bp }\end{array}$ & TIM1ef+G & $\begin{array}{l}\text { Carbone \& } \\
\text { Kohn (1999) }\end{array}$ \\
\hline TUB2 & $\beta$-Tubulin & $\begin{array}{l}\text { BT-2F } \\
\text { BT-4R }\end{array}$ & $\begin{array}{l}\text { AAC ATG CGT GAG ATT GTA AGT } \\
\text { TAG TGA CCC TTG GCC CAG TTG }\end{array}$ & $\begin{array}{l}\text { Colletotrichum } \\
: 479 \mathrm{bp} \\
\text { Didymellaceae } \\
: 338 \mathrm{bp}\end{array}$ & $\begin{array}{l}\text { Colletotrichum } \\
\text { : TrNef }+\mathrm{G} \\
\text { Didymellaceae } \\
: \text { TPM2uf }+\mathrm{I}+\mathrm{G}\end{array}$ & $\begin{array}{l}\text { O’Donnell \& } \\
\text { Cigelnik (1997) }\end{array}$ \\
\hline
\end{tabular}

Table 2 GenBank accession numbers of the nucleotide sequences of Alternaria species used in this study

\begin{tabular}{|c|c|c|c|c|c|c|}
\hline \multirow[t]{2}{*}{ Species } & \multirow{2}{*}{$\begin{array}{l}\text { Culture Collection } \\
\text { number }{ }^{1}\end{array}$} & \multicolumn{5}{|c|}{ GenBank Accession Numbers ${ }^{3}$} \\
\hline & & ITS & GAPDH & RPB2 & TEF1- $\alpha$ & Alt-a 1 \\
\hline Alternaria alstroemeriae & CBS 118808 & KP124296 & KP124153 & KP124764 & KP125071 & KP123845 \\
\hline Alternaria alstroemeriae & CBS 118809 & KP124297 & KP124154 & KP124765 & KP125072 & - \\
\hline Alternaria alternantherae & CBS 124392 & KC584179 & KC584096 & KC584374 & KC584633 & KP123846 \\
\hline Alternaria alternata & CBS 174.52 & KC584228 & KC584152 & DQ677964 & KC584704 & KP123856 \\
\hline Alternaria alternata & CBS 175.52 & KC584227 & KC584151 & KC584445 & KC584703 & KP123857 \\
\hline Alternaria alternata & CBS $916.96^{\mathrm{T}}$ & AF347031 & AY278808 & KC584375 & KC584634 & AY563301 \\
\hline Alternaria alternata & CBS 102595 & FJ266476 & AY562411 & KC584408 & KC584666 & AY563306 \\
\hline Alternaria alternata & CBS 118812 & KC584193 & KC584112 & KC584393 & KC584652 & KP123905 \\
\hline Alternaria alternata & JZB3180002 & MH827031 & MH853645 & MH853718 & MH853703 & MH853692 \\
\hline Alternaria alternata & MFLUCC 18-1587 & MH827038 & MH853652 & MH853725 & MH853710 & MH853699 \\
\hline Alternaria alternata & JZB3180011 & MH827040 & MH853654 & MH853727 & MH853712 & MH853701 \\
\hline Alternaria alternata & JZB3180012 & MH827041 & MH853655 & MH853728 & MH853713 & - \\
\hline Alternaria alternata & JZB3180014 & MH827043 & MH853657 & MH853730 & MH853715 & - \\
\hline Alternaria alternata & MFLUCC 18-1586 & MH827044 & MH853658 & MH853731 & MH853716 & - \\
\hline Alternaria anigozanthi & CBS $121920^{\mathrm{T}}$ & KC584180 & KC584097 & KC584376 & KC584635 & - \\
\hline Alternaria arborescens & CBS $102605^{\mathrm{T}}$ & AF347033 & AY278810 & KC584377 & KC584636 & AY563303 \\
\hline Alternaria arborescens & CBS 101.13 & KP124392 & KP124244 & KP124862 & KP125170 & KP123940 \\
\hline
\end{tabular}


Table 2 Continued.

\begin{tabular}{|c|c|c|c|c|c|c|}
\hline \multirow[t]{2}{*}{ Species } & \multirow{2}{*}{$\begin{array}{l}\text { Culture Collection } \\
\text { number }{ }^{1}\end{array}$} & \multicolumn{5}{|c|}{ GenBank Accession Numbers ${ }^{3}$} \\
\hline & & ITS & GAPDH & RPB2 & TEF1- $\alpha$ & Alt-a 1 \\
\hline Alternaria arborescens & CBS 112633 & KP124400 & KP124252 & KP124870 & KP125178 & KP123947 \\
\hline Alternaria aspera & CBS $115269^{\mathrm{T}}$ & KC584242 & KC584166 & KC584474 & KC584734 & KF533899 \\
\hline Alternaria betae-kenyensis & CBS $118810^{\mathrm{T}}$ & KP124419 & KP124270 & KP124888 & KP125197 & KP123966 \\
\hline Alternaria brassicicola & CBS 118699 & JX499031 & KC584103 & KC584383 & KC584642 & - \\
\hline Alternaria burnsii & CBS 108.27 & KC584236 & KC584162 & KC584468 & KC584727 & KP123850 \\
\hline Alternaria burnsii & CBS $107.38^{\mathrm{T}}$ & KP124420 & JC646305 & KP124889 & KP125198 & KP123967 \\
\hline Alternaria carotiincultae & CBS $109381^{\mathrm{T}}$ & KC584188 & KC584106 & KC584386 & KC584645 & - \\
\hline Alternaria cheiranthi & CBS 109384 & AF229457 & KC584107 & KC584387 & KC584646 & JQ905106 \\
\hline Alternaria cinerariae & CBS 116495 & KC584190 & KC584109 & КC584389 & КС584648 & - \\
\hline Alternaria cucurbitae & CBS 483.81 & FJ266483 & AY562418 & KC584483 & KC584743 & - \\
\hline Alternaria dauci & CBS 117097 & KC584192 & KC584111 & KC584392 & KC584651 & KJ718678 \\
\hline Alternaria dianthicola & CBS 116491 & KC584194 & KC584113 & КC584394 & КC584653 & - \\
\hline Alternaria eichhorniae & CBS $489.92^{\mathrm{T}}$ & KC146356 & KP124276 & KP124895 & KP125204 & KP123973 \\
\hline Alternaria eichhorniae & CBS 119778 & KP124426 & KP124277 & KP124896 & KP125205 & - \\
\hline Alternaria gaisen & CBS 632.93 & KC584197 & KC584116 & КС584399 & KC584658 & KP123974 \\
\hline Alternaria gaisen & CBS 118488 & KP124427 & KP124278 & KP125206 & KP124897 & KP123975 \\
\hline Alternaria gossypina & CBS 100.23 & KP124429 & KP124280 & KP124899 & KP125208 & KP123977 \\
\hline Alternaria gossypina & CBS $104.32^{\mathrm{T}}$ & KP124430 & JQ646312 & KP124900 & KP125209 & JQ646395 \\
\hline Alternaria gypsophilae & CBS $107.41^{\mathrm{T}}$ & KC584199 & KC584118 & KC584401 & KC584660 & JQ646304 \\
\hline Alternaria iridiaustralis & CBS $118486^{\mathrm{T}}$ & KP124435 & KP124284 & KP124905 & KP125214 & KP123981 \\
\hline Alternaria iridiaustralis & CBS 118404 & KP124434 & KP124283 & KP124904 & KP125213 & KP123980 \\
\hline Alternaria jacinthicola & CBS $133751^{\mathrm{T}}$ & KP124438 & KP124287 & KP124908 & KP125217 & KP123984 \\
\hline Alternaria jacinthicola & CPC 25267 & KP124439 & KP124288 & KP124909 & KP125218 & KP123985 \\
\hline Alternaria japonica & CBS 118390 & KC584201 & KC584121 & KC584405 & KC584663 & - \\
\hline Alternaria juxtiseptata & CBS $119673^{\mathrm{T}}$ & KC584202 & KC584122 & KC584406 & KC584664 & - \\
\hline Alternaria leucanthemi & CBS $421.65^{\mathrm{T}}$ & KC584240 & KC584164 & KC584472 & KC584732 & - \\
\hline Alternaria longipes & CBS 540.94 & AY278835 & AY278811 & KC584409 & KC584667 & AY563304 \\
\hline Alternaria longipes & CBS 917.96 & KP124442 & KP124291 & KP124912 & KP125226 & KP123988 \\
\hline Alternaria longipes & CBS 121333 & KP124444 & KP124293 & KP124914 & KP125223 & KP123990 \\
\hline Alternaria macrospora & CBS $117228^{\mathrm{T}}$ & KC584204 & KC584124 & KC584410 & KC584668 & KJ718702 \\
\hline
\end{tabular}


Table 2 Continued.

\begin{tabular}{|c|c|c|c|c|c|c|}
\hline \multirow[t]{2}{*}{ Species } & \multirow{2}{*}{$\begin{array}{l}\text { Culture Collection } \\
\text { number }{ }^{1}\end{array}$} & \multicolumn{5}{|c|}{ GenBank Accession Numbers ${ }^{3}$} \\
\hline & & ITS & GAPDH & RPB2 & TEF1- $\alpha$ & Alt-a 1 \\
\hline Alternaria nepalensis & CBS $118700^{\mathrm{T}}$ & KC584207 & KC584126 & KC584414 & KC584672 & - \\
\hline Alternaria nobilis & CBS 116490 & KC584208 & KC584127 & KC584415 & KC584673 & JQ646385 \\
\hline Alternaria obovoidea & CBS 101229 & FJ266487 & FJ266498 & KC584485 & KC584745 & FJ266513 \\
\hline Alternaria panax & CBS 482.81 & KC584209 & KC584128 & KC584417 & KC584675 & - \\
\hline Alternaria perpunctulata & CBS $115267^{\mathrm{T}}$ & KC584210 & KC584129 & KC584418 & KC584676 & JQ905111 \\
\hline Alternaria photistica & CBS $212.86^{\mathrm{T}}$ & KC584212 & KC584131 & KC584420 & KC584678 & - \\
\hline Alternaria porri & CBS 116698 & DQ323700 & KC584132 & KC584421 & KC584679 & KJ718726 \\
\hline Alternaria prunicola & MFLUCC 18-1598 & MH827032 & MH853646 & MH853719 & MH853704 & MH853693 \\
\hline Alternaria prunicola & MFLUCC 18-1596 & MH827033 & MH853647 & MH853720 & MH853705 & MH853694 \\
\hline Alternaria prunicola & JZB3180005 & MH827034 & MH853648 & MH853721 & MH853706 & MH853695 \\
\hline Alternaria prunicola & JZB3180006 & MH827035 & MH853649 & MH853722 & MH853707 & MH853696 \\
\hline Alternaria prunicola & MFLUCC 18-1597 ${ }^{\mathrm{T}}$ & MH827036 & MH853650 & MH853723 & MH853708 & MH853697 \\
\hline Alternaria prunicola & MFLUCC 18-1599 & MH827037 & MH853651 & MH853724 & MH853709 & MH853698 \\
\hline Alternaria prunicola & JZB3180013 & MH827042 & MH853656 & MH853729 & MH853714 & - \\
\hline Alternaria pseudoeichhorniae & MFLUCC 18-1589 ${ }^{\mathrm{T}}$ & MH827030 & MH853644 & MH853717 & MH853702 & - \\
\hline Alternaria pseudorostrata & CBS $119411^{\mathrm{T}}$ & JN383483 & AY562406 & KC584422 & KC584680 & - \\
\hline Alternaria radicina & CBS $245.67^{\mathrm{T}}$ & KC584213 & KC584133 & KC584423 & KC584681 & FN689405 \\
\hline Alternaria saponariae & CBS 116492 & KC584215 & KC584135 & KC584425 & KC584683 & - \\
\hline Alternaria septospora & CBS 109.38 & FJ266489 & FJ266500 & KC584487 & KC584747 & FJ266515 \\
\hline Alternaria simsimi & CBS $115265^{\mathrm{T}}$ & JF780937 & KC584137 & KC584428 & KC584686 & JQ905110 \\
\hline Alternaria solani & CBS 116651 & KC584217 & KC584139 & KC584430 & KC584688 & - \\
\hline Alternaria sonchi & CBS 119675 & KC584220 & KC584142 & KC584433 & KC584691 & - \\
\hline Alternaria sp. & CBS 115.44 & KC584214 & KC584134 & KC584424 & KC584682 & - \\
\hline Alternaria tagetica & CBS 479.81 & KC584221 & KC584143 & KC584434 & KC584692 & KJ718761 \\
\hline Alternaria tenuissima & CBS 918.96 & AF347032 & AY278809 & KC584435 & KC584693 & AY563302 \\
\hline Alternaria terricola & CBS $202.67^{\mathrm{T}}$ & FJ266490 & KC584177 & KC584490 & KC584750 & FJ266516 \\
\hline Alternaria tomato & CBS 103.30 & KP124445 & KP124294 & KP124915 & KP125224 & KP123991 \\
\hline Alternaria tomato & CBS 114.35 & KP124446 & KP124295 & KP124916 & KP125225 & KP123992 \\
\hline Alternaria vaccariicola & CBS $118714^{\mathrm{T}}$ & KC584224 & KC584147 & KC584439 & KC584697 & - \\
\hline
\end{tabular}


${ }^{1}$ CBS: Culture collection of the Centraalbureau voor Schimmelcultures, Fungal Biodiversity Centre, Utrecht, The Netherlands; CPC: Personal collection of P.W. Crous, Utrecht, The Netherlands; JZB: Beijing Academy of Agriculture and Forestry Sciences culture collection, Beijing, China; MFLUCC: Mae Fah Luang University Culture Collection, Thailand;

Ex-type, neo-type and epi-type cultures are marked with superscript ${ }^{\mathrm{T}}$ and newly generated sequences are shown in bold face.

ITS: internal transcribed spacer regions 1 \& 2 including 5.8S nrDNA gene; GAPDH: glyceraldehyde-3-phosphate dehydrogenase; RPB2: RNA polymerase II second subunit; TEF 1- $\alpha$ : Partial translation elongation factor 1- $\alpha$; Alt-a 1: Alternaria major allergen 1.

Table 3 GenBank accession numbers of the nucleotide sequences of Colletotrichum species used in this study.

\begin{tabular}{|c|c|c|c|c|c|c|}
\hline \multirow{2}{*}{ Species } & \multirow{2}{*}{$\begin{array}{l}\text { Culture Collection } \\
\text { number }\end{array}$} & \multicolumn{5}{|c|}{ GenBank Accession Numbers ${ }^{3}$} \\
\hline & & ITS & GAPDH & CHS-1 & ACT & TUB \\
\hline Colletotrichum aenigma & ICMP $18608^{\mathrm{T}}$ & JX010244 & JX010044 & JX009774 & JX009443 & JX010389 \\
\hline Colletotrichum aenigma & ICMP 18686 & JX010243 & JX009913 & JX009789 & JX009519 & JX010390 \\
\hline Colletotrichum aenigma & MFLUCC 18-1604 & MH817393 & MH853673 & MH853676 & MH853679 & MH853682 \\
\hline Colletotrichum aenigma & MFLUCC 18-1603 & MH817394 & MH853674 & MH853677 & MH853680 & MH853683 \\
\hline Colletotrichum aeschynomenes & ICMP $17673^{\mathrm{T}}$ & JX010176 & JX009930 & JX009799 & JX009483 & JX010392 \\
\hline Colletotrichum alatae & ICMP $17919^{\mathrm{T}}$ & JX010190 & JX009990 & JX009837 & JX009471 & JX010383 \\
\hline Colletotrichum alatae & ICMP 18122 & JX010191 & JX010011 & JX009846 & JX009470 & JX010449 \\
\hline Colletotrichum alienum & ICMP $12071^{\mathrm{T}}$ & JX010251 & JX010028 & JX009882 & JX009572 & JX010411 \\
\hline Colletotrichum alienum & ICMP 18691 & X010217 & JX010018 & JX009754 & JX009580 & JX010385 \\
\hline Colletotrichum aotearoa & ICMP $18537^{\mathrm{T}}$ & X010205 & JX010005 & JX009853 & JX009564 & JX010420 \\
\hline Colletotrichum aotearoa & & & & & & 0417 \\
\hline Colletotrichum asianum & ICMP & & & & & 406 \\
\hline Colletotrichum asianum & ICMP & & & JXOC & JX0 & 0384 \\
\hline Colletotrichum boninense & CBS $123755^{\mathrm{T}}$ & 5153 & JQ005240 & JQ005327 & JQ005501 & JQ005588 \\
\hline Colletotrichum camelliae & CGMCC $3.14925^{\mathrm{T}}$ & KJ955081 & KJ954782 & - & KJ954363 & KJ955230 \\
\hline Colletotrichum chengpingense & MFLUCC $15-0022^{\mathrm{T}}$ & KP683152 & KP852469 & KP852449 & KP683093 & KP852490 \\
\hline Colletotrichum clidemiae & ICMP $18658^{\mathrm{T}}$ & JX010265 & JX009989 & JX009877 & JX009537 & JX010438 \\
\hline Colletotrichum conoides & CAUG17 ${ }^{\mathrm{T}}$ & KP890168 & KP890162 & KP890156 & KP890144 & KP890174 \\
\hline Colletotrichum cordylinicola & ICMP $18579^{\mathrm{T}}$ & JX010226 & JX009975 & JX009864 & HM470235 & JX010440 \\
\hline Colletotrichum endophytica & $\mathrm{LC} 0324$ & KC633854 & KC832854 & - & KF306258 & - \\
\hline Colletotrichum fructicola & ICMP $18581^{\mathrm{T}}$ & JX010165 & JX010033 & JX009873 & FJ907426 & JX010405 \\
\hline Colletotrichum fructicola & ICMP 18645 & JX010172 & JX009992 & JX009866 & JX009543 & JX010408 \\
\hline Colletotrichum fructivorum & Coll1414 $4^{\mathrm{T}}$ & $\mathrm{JX} 145145$ & & & & JX145196 \\
\hline
\end{tabular}


Table 3 Continued.

\begin{tabular}{|c|c|c|c|c|c|c|}
\hline \multirow{2}{*}{ Species } & \multirow{2}{*}{$\begin{array}{l}\text { Culture Collection } \\
\text { number }\end{array}$} & \multicolumn{5}{|c|}{ GenBank Accession Numbers ${ }^{3}$} \\
\hline & & ITS & GAPDH & CHS-1 & $\mathbf{A C T}$ & TUB \\
\hline Colletotrichum gloeosporioides & CBS $112999^{\mathrm{T}}$ & JQ005152 & JQ005239 & JQ005326 & JQ005500 & JQ005587 \\
\hline Colletotrichum gloeosporioides & ICMP 18697 & JX010154 & JX009987 & JX009780 & JX009557 & - \\
\hline Colletotrichum gloeosporioides & ICMP 19121 & JX010148 & JX010054 & JX009903 & JX009558 & - \\
\hline Colletotrichum grevilleae & CBS $132879^{\mathrm{T}}$ & KC297078 & KC297010 & KC296987 & KC296941 & KC297102 \\
\hline Colletotrichum grossum & CAUG7 ${ }^{\mathrm{T}}$ & KP890165 & KP890159 & KP890153 & KP890153 & KP890171 \\
\hline Colletotrichum hebeiense & MFLUCC13-0726 ${ }^{\mathrm{T}}$ & KF156863 & KF377495 & KF289008 & KF377532 & KF288975 \\
\hline Colletotrichum hebeiense & MFLUCC14-1213 ${ }^{\mathrm{T}}$ & KF156873 & KF377505 & - & KF377542 & - \\
\hline Colletotrichum henanense & CGMCC $3.17354^{\mathrm{T}}$ & KJ955109 & KJ954810 & - & KM023257 & KJ955257 \\
\hline Colletotrichum horii & ICMP $10492^{\mathrm{T}}$ & GQ329690 & GQ329681 & JX009752 & JX009438 & JX010450 \\
\hline Colletotrichum horii & ICMP 17968 & JX010212 & GQ329682 & JX009811 & JX009547 & JX010378 \\
\hline Colletotrichum jiangxiense & CGMCC $3.17363^{\mathrm{T}}$ & KJ955201 & KJ954902 & - & KJ954471 & KJ955348 \\
\hline Colletotrichum kahawae & ICMP $17816^{\mathrm{T}}$ & JX010231 & JX010046 & JX009813 & JX009452 & JX010444 \\
\hline Colletotrichum kahawae & ICMP 17905 & JX010232 & JX010012 & JX009816 & JX009561 & JX010431 \\
\hline Colletotrichum musae & ICMP $19119^{\mathrm{T}}$ & JX010146 & JX010050 & JX009896 & JX009433 & HQ596280 \\
\hline Colletotrichum musae & ICMP 17817 & JX010142 & JX010015 & JX009815 & JX009432 & JX010395 \\
\hline Colletotrichum nupharicola & ICMP $18187^{\mathrm{T}}$ & JX010187 & JX009972 & JX009835 & JX009437 & JX010398 \\
\hline Colletotrichum nupharicola & ICMP 17938 & JX010189 & JX009936 & JX009834 & JX009486 & JX010397 \\
\hline Colletotrichum pandanicola & MFLUCC $17-0571^{\mathrm{T}}$ & MG646967 & MG646934 & MG646931 & MG646938 & MG646926 \\
\hline Colletotrichum proteae & CBS $132882^{\mathrm{T}}$ & KC297079 & KC297009 & KC296986 & KC296940 & KC297101 \\
\hline Colletotrichum psidii & ICMP $19120^{\mathrm{T}}$ & JX010219 & JX009967 & JX009901 & JX009515 & JX010443 \\
\hline Colletotrichum queenslandicum & ICMP $1778^{\mathrm{T}}$ & JX010276 & JX009934 & JX009899 & JX009447 & JX010414 \\
\hline Colletotrichum rhexiae & Coll 1026 & JX145128 & - & - & - & JX145179 \\
\hline Colletotrichum salsolae & ICMP 19051 & JX010242 & JX009916 & JX009863 & JX009562 & JX010403 \\
\hline Colletotrichum siamense & ICMP 18578 & JX010171 & JX009924 & JX009865 & FJ907423 & JX010404 \\
\hline Colletotrichum siamense & ICMP 18574 & JX010270 & JX010002 & JX009798 & JX009535 & JX010391 \\
\hline Colletotrichum syzygicola & MFLUCC $10-0624^{\mathrm{T}}$ & KF242094 & KF242156 & - & KF157801 & KF254880 \\
\hline Colletotrichum temperatum & Coll883 T & JX145159 & - & - & - & JX145211 \\
\hline Colletotrichum theobromicola & ICMP $18649^{\mathrm{T}}$ & JX010294 & JX010006 & JX009869 & JX009444 & JX010447 \\
\hline Colletotrichum theobromicola & ICMP 17895 & JX010284 & JX010057 & JX009828 & JX009568 & JX010382 \\
\hline Colletotrichum theobromicola & ICMP 17958 & JX010291 & JX009948 & JX009822 & JX009498 & JX010381 \\
\hline
\end{tabular}


Table 3 Continued.

\begin{tabular}{|c|c|c|c|c|c|c|}
\hline \multirow{2}{*}{ Species } & \multirow{2}{*}{$\begin{array}{l}\text { Culture Collection } \\
\text { number }{ }^{1}\end{array}$} & \multicolumn{5}{|c|}{ GenBank Accession Numbers ${ }^{3}$} \\
\hline & & ITS & GAPDH & CHS-1 & ACT & TUB \\
\hline Colletotrichum theobromicola & ICMP 17927 & JX010286 & JX010024 & JX009830 & JX009516 & JX010373 \\
\hline Colletotrichum theobromicola & ICMP 17957 & JX010289 & JX009962 & JX009821 & JX009575 & JX010380 \\
\hline $\begin{array}{l}\text { Colletotrichum } \\
\text { pseudotheobromicola }\end{array}$ & MFLUCC 18-1602 ${ }^{\mathrm{T}}$ & MH817395 & MH853675 & MH853678 & MH853681 & MH853684 \\
\hline Colletotrichum ti & ICMP $4832^{\mathrm{T}}$ & JX010269 & JX009952 & JX009898 & JX009520 & JX010442 \\
\hline Colletotrichum tropicale & CBS $124949^{\mathrm{T}}$ & JX010264 & JX010007 & JX009870 & JX009489 & JX010407 \\
\hline Colletotrichum viniferum & GZAAS5.08601 ${ }^{\mathrm{T}}$ & JN412804 & JN412798 & - & JN412795 & JN412813 \\
\hline Colletotrichum viniferum & GZAAS5.08608 & JN412802 & JN412800 & - & JN412793 & JN412811 \\
\hline Colletotrichum viniferum & GZAAS5.08614 & JN412807 & JN412799 & - & JN412790 & JN412809 \\
\hline Colletotrichum wuxiense & CGMCC $3.17894^{\mathrm{T}}$ & KU251591 & KU252045 & KU251939 & KU251672 & KU252200 \\
\hline Colletotrichum xanthorrhoeae & ICMP $17903^{\mathrm{T}}$ & JX010261 & JX009927 & JX009823 & - & JX010448 \\
\hline
\end{tabular}

${ }^{1}$ CBS: Culture collection of the Centraalbureau voor Schimmelcultures, Fungal Biodiversity Centre, Utrecht, The Netherlands; CGMCC: China General Microbiological Culture Collection Center, Institute of Microbiology, Chinese Academy of Sciences, China; GZAAS: Guizhou Academy of Agricultural Sciences Herbarium, China; JZB: Beijing Academy of Agriculture and Forestry Sciences culture collection, Beijing, China; ICMP: International Collection of Microorganisms for Plants, Landcare Research, New Zealand; LC: Laboratory of Cryptogamy, National Museum of Natural History, Paris, France; MFLUCC: Mae Fah Luang University Culture Collection, Chiang Rai, Thailand.

${ }^{2}$ Ex-type, neo-type and epi-type cultures are marked with superscript ${ }^{\mathrm{T}}$ and newly generated sequences are shown in bold face.

${ }^{3}$ ITS: internal transcribed spacer regions $1 \& 2$ including 5.8S nrDNA gene; GAPDH: glyceraldehyde-3-phosphate dehydrogenase; TUB: ß-tubulin; CHS-1: Chitin synthase; ACT: Actin.

Table 4 GenBank accession numbers of the nucleotide sequences of Didymellaceae species used in this study.

\begin{tabular}{|c|c|c|c|c|c|}
\hline \multirow{2}{*}{ Species $^{1}$} & \multirow{2}{*}{$\begin{array}{l}\text { Culture Collection } \\
\text { number }\end{array}$} & \multicolumn{4}{|c|}{ GenBank Accession Numbers ${ }^{3}$} \\
\hline & & LSU & ITS & TUB & RPB2 \\
\hline Epicoccum brasiliense & CBS $120105^{\mathrm{T}}$ & GU238049 & GU237760 & GU237588 & KT389627 \\
\hline Epicoccum camelliae & CGMCC $3.18343^{\mathrm{T}}$ & KY742245 & KY742091 & KY742333 & KY742170 \\
\hline Epicoccum camelliae & UTHSC:DI16-201 & LN907344 & LT592902 & LT592971 & LT593040 \\
\hline Epicoccum camelliae & UTHSC:DI16-202 & LN907345 & LT592903 & LT592972 & LT593041 \\
\hline Epicoccum camelliae & UTHSC:DI16-206 & LN907349 & LT592906 & LT592975 & LT593044 \\
\hline Epicoccum camelliae & UTHSC:DI16-280 & LN907423 & LT592937 & LT593006 & LT593076 \\
\hline
\end{tabular}


Table 4 Continued.

\begin{tabular}{|c|c|c|c|c|c|}
\hline \multirow{2}{*}{ Species ${ }^{1}$} & \multirow{2}{*}{$\begin{array}{ll}\text { Culture } & \text { Collection } \\
\text { number }\end{array}$} & \multicolumn{4}{|c|}{ GenBank Accession Numbers ${ }^{3}$} \\
\hline & & LSU & ITS & TUB & RPB2 \\
\hline Epicoccum camelliae & UTHSC:DI16-338 & LN907481 & LT592959 & LT593028 & LT593098 \\
\hline Epicoccum camelliae & UTHSC:DI16-345 & LN907488 & LT592961 & LT593030 & LT593100 \\
\hline Epicoccum camelliae & LC 4862 & KY742246 & KY742092 & KY742334 & KY742171 \\
\hline Epicoccum catenisporum & CBS $181.80^{\mathrm{T}}$ & LT623213 & FJ427069 & FJ427175 & LT623253 \\
\hline Epicoccum cedri & MFLUCC $17-1058^{\mathrm{T}}$ & KY711172 & KY711170 & KY711168 & - \\
\hline Epicoccum dendrobii & CGMCC $3.18359^{\mathrm{T}}$ & KY742247 & KY742093 & KY742335 & - \\
\hline Epicoccum dendrobii & LC 8146 & KY742248 & KY74209 & KY742336 & - \\
\hline Epicoccum draconis & CBS 186.83 & GU238070 & GU237795 & GU237607 & KT389628 \\
\hline Epicoccum duchesneae & LC 8147 & KY742250 & KY742096 & KY742338 & - \\
\hline Epicoccum duchesneae & CGMCC $3.18345^{\mathrm{T}}$ & KY742249 & KY742095 & KY742337 & - \\
\hline Epicoccum henningsii & CBS 104.80 & GU238081 & GU237731 & GU237612 & KT389629 \\
\hline Epicoccum hordei & CGMCC $3.18360^{\mathrm{T}}$ & KY742251 & KY742097 & KY742339 & - \\
\hline Epicoccum hordei & LC 8149 & KY742252 & KY742098 & KY742340 & - \\
\hline Epicoccum huancayense & CBS $105.80^{\mathrm{T}}$ & GU238084 & GU237732 & GU237615 & KT389630 \\
\hline Epicoccum italicum & CGMCC $3.18361^{\mathrm{T}}$ & KY742253 & KY742099 & KY742341 & KY742172 \\
\hline Epicoccum italicum & LC 8151 & KY74225 & KY742100 & KY742342 & KY742173 \\
\hline Epicoccum keratinophilum & UTHSC:DI16-244 & LN907387 & LT592924 & LT592993 & LT593062 \\
\hline Epicoccum keratinophilum & UTHSC:DI16-258 & LN907401 & LT592928 & LT592997 & LT593066 \\
\hline Epicoccum keratinophilum & UTHSC:DI16-271 ${ }^{\mathrm{T}}$ & LN907414 & LT592930 & LT592999 & LT593068 \\
\hline Epicoccum keratinophilum & UTHSC:DI16-272 & LN907415 & LT592931 & LT593000 & LT593069 \\
\hline Epicoccum keratinophilum & UTHSC:DI16-299 & LN907442 & LT592947 & LT593016 & LT593086 \\
\hline Epicoccum latusicollum & UTHSC:DI16-197 & LT907340 & LT592898 & LT592967 & LT593036 \\
\hline Epicoccum latusicollum & CGMCC $3.18346^{\mathrm{T}}$ & KY742255 & KY742101 & KY742343 & KY742174 \\
\hline Epicoccum latusicollum & LC 4859 & KY742256 & KY742102 & KY742344 & KY742175 \\
\hline Epicoccum layuense & CGMCC $3.18362^{\mathrm{T}}$ & KY742261 & KY742107 & KY742349 & - \\
\hline Epicoccum layuense & LC 8156 & KY742262 & KY742108 & KY742350 & - \\
\hline Epicoccum mackenziei & MFLUCC $16-0335^{\mathrm{T}}$ & KX698028 & KX698039 & KX698032 & KX698035 \\
\hline Epicoccum nigrum & CBS 125.82 & GU237974 & FJ426995 & FJ427106 & КT389631 \\
\hline Epicoccum nigrum & CBS $173.73^{\mathrm{T}}$ & GU237975 & FJ426996 & FJ427107 & KT389632 \\
\hline Epicoccum ovisporum & CBS $180.80^{\mathrm{T}}$ & LT623212 & FJ427068 & FJ427174 & LT623252 \\
\hline
\end{tabular}


Table 4 Continued.

\begin{tabular}{|c|c|c|c|c|c|}
\hline \multirow{2}{*}{ Species ${ }^{1}$} & \multirow{2}{*}{$\begin{array}{l}\text { Culture Collection } \\
\text { number }\end{array}$} & \multicolumn{4}{|c|}{ GenBank Accession Numbers ${ }^{3}$} \\
\hline & & LSU & ITS & TUB & RPB2 \\
\hline Epicoccum pimprinum & PD 77/1028 & GU237977 & FJ427050 & FJ427160 & KT389633 \\
\hline Epicoccum plurivorum & CBS $558.81^{\mathrm{T}}$ & GU238132 & GU237888 & GU237647 & KT389634 \\
\hline Epicoccum pneumoniae & UTHSC:DI16-257 ${ }^{\mathrm{T}}$ & LN907400 & LT592927 & LT592996 & LT593065 \\
\hline Epicoccum poaceicola & MFLUCC $15-0448^{\mathrm{T}}$ & KX954396 & KX965727 & KY197980 & - \\
\hline Eрісоссит роае & LC 8161 & KY742268 & KY742114 & KY742356 & KY742183 \\
\hline Epicocсит роае & CGMCC $3.18363^{\mathrm{T}}$ & KY742267 & KY742113 & KY742355 & KY742182 \\
\hline Epicoccum poae & LC 8162 & KY742269 & KY742115 & KY742357 & KY742184 \\
\hline Epicoccum proteae & CBS $114179^{\mathrm{T}}$ & JQ044452 & JQ044433 & LT623230 & LT623251 \\
\hline Epicoccum pruni & MFLUCC 17-1059 & KY711171 & KY711169 & KY711167 & KY711173 \\
\hline Epicoccum pseudokeratinophilum & MFLUCC 18-1593 ${ }^{\mathrm{T}}$ & - & MH827002 & MH853666 & MH853659 \\
\hline Epicoccum rosae & MFLUCC 15-3639 & MG829009 & MG828899 & - & - \\
\hline Epicoccum sorghinum & CBS 179.80 & GU237978 & FJ427067 & FJ427173 & KT389635 \\
\hline Epicoccum sorghinum & CBS 627.68 & GU237979 & FJ427072 & FJ427178 & KT389636 \\
\hline Epicoccum sorghinum & UTHSC:DI16-288 & LN907431 & LT592940 & LT593009 & LT593079 \\
\hline Epicoccum sorghinum & UTHSC:DI16-301 & LN907444 & LT592948 & LT593017 & LT593087 \\
\hline Epicoccum thailandicum & MFLUCC $16-0892^{\mathrm{T}}$ & KY703620 & KY703619 & - & - \\
\hline Epicoccum tritici & MFLUCC 16-0277 & KX954391 & KX926426 & KY197979 & - \\
\hline Epicoccum viticis & BRIP 29294 & KY742271 & KY742117 & KY742359 & - \\
\hline Epicoccum viticis & CGMCC $3.18344^{\mathrm{T}}$ & KY742272 & KY742118 & KY742360 & KY742186 \\
\hline Neocucurbitaria aquatica & CBS $297.74^{\mathrm{T}}$ & EU754177 & LT623221 & LT623238 & LT623278 \\
\hline Nothophoma anigozanthi & CBS $381.91^{\mathrm{T}}$ & GU238039 & GU237852 & GU237580 & KT389655 \\
\hline Nothophoma arachidis-hypogaeae & CBS 125.93 & GU238043 & GU237771 & GU237583 & KT389656 \\
\hline Nothophoma gossypiicola & CBS 377.67 & GU238079 & GU237845 & GU237611 & KT389658 \\
\hline Nothophoma gossypiicola & UTHSC:DI16-294 & LN907437 & LT592943 & LT593012 & LT593082 \\
\hline Nothophoma infossa & CBS $123395^{\mathrm{T}}$ & GU238089 & FJ427025 & FJ427135 & KT389659 \\
\hline Nothophoma macrospora & CBS $140674^{\mathrm{T}}$ & LN880537 & LN880536 & LN880539 & LT593073 \\
\hline Nothophoma multilocularis & AUMC- $12003^{\mathrm{T}}$ & KY996744 & - & - & - \\
\hline Nothophoma pruni & JZB380015 & MH827025 & MH827004 & MH853668 & MH853661 \\
\hline Nothophoma pruni & MFLUCC 18-1601 & MH827026 & MH827005 & MH853669 & MH853662 \\
\hline Nothophoma pruni & JZB380017 & MH827027 & MH827006 & MH853670 & MH853663 \\
\hline
\end{tabular}


Table 4 Continued.

\begin{tabular}{|c|c|c|c|c|c|}
\hline \multirow{2}{*}{ Species ${ }^{1}$} & \multirow{2}{*}{$\begin{array}{l}\text { Culture Collection } \\
\text { number }\end{array}$} & \multicolumn{4}{|c|}{ GenBank Accession Numbers ${ }^{3}$} \\
\hline & & LSU & ITS & TUB & RPB2 \\
\hline Nothophoma pruni & MFLUCC 18-1600 $^{\mathrm{T}}$ & MH827028 & MH827007 & MH853671 & MH853664 \\
\hline Nothophoma quercina & CBS 633.92 & EU754127 & GU237900 & GU237609 & KT389657 \\
\hline Nothophoma quercina & UTHSC:DI16-270 & LN907413 & LT592929 & LT592998 & LT593067 \\
\hline Nothophoma quercina & MFLUCC 18-1588 & MH827029 & MH827008 & MH853672 & MH853665 \\
\hline Nothophoma raii & MCC $1082^{\mathrm{T}}$ & - & MF664467 & MF664468 & - \\
\hline Nothophoma variabilis & UTHSC:DI16-285 ${ }^{\mathrm{T}}$ & LN907428 & LT592939 & LT593008 & LT593078 \\
\hline Stagonosporopsis actaeae & CBS $106.96^{\mathrm{T}}$ & GU238166 & GU237734 & KT389672 & GU237671 \\
\hline Stagonosporopsis actaeae & CBS 114303 & KT389760 & KT389544 & - & KT389847 \\
\hline Stagonosporopsis ajacis & CBS $177.93^{\mathrm{T}}$ & GU238168 & GU237791 & KT389673 & GU237673 \\
\hline Stagonosporopsis alianthicola & MFLUCC16-1439 ${ }^{\mathrm{T}}$ & - & KY100872 & KY100878 & KY100876 \\
\hline Stagonosporopsis andigena & CBS 101.80 & GU238169 & GU237714 & - & GU237674 \\
\hline Stagonosporopsis andigena & CBS 269.80 & GU238170 & GU237817 & - & GU237675 \\
\hline Stagonosporopsis artemisiicola & CBS 102636 & GU238171 & GU237728 & KT389674 & GU237676 \\
\hline Stagonosporopsis astragali & CBS 178.25 & GU238172 & GU237792 & - & GU237677 \\
\hline Stagonosporopsis bomiensis & $\mathrm{LC} 8167^{\mathrm{T}}$ & KY742277 & KY742123 & KY742189 & KY742365 \\
\hline Stagonosporopsis bomiensis & LC 8168 & KY742278 & KY742124 & KY742190 & KY742366 \\
\hline Stagonosporopsis caricae & CBS 248.90 & GU238175 & GU237807 & - & GU237680 \\
\hline Stagonosporopsis caricae & CBS 282.76 & GU238177 & GU237821 & - & GU237682 \\
\hline Stagonosporopsis centaureae & MFLUCC 16-0787 & KX611238 & KX611240 & - & - \\
\hline Stagonosporopsis citrulli & ATCC TSD- $2^{\mathrm{T}}$ & - & KJ855546 & KJ855602 & - \\
\hline Stagonosporopsis citrulli & MFLUCC 18-1595 & MH827024 & MH827003 & MH853667 & MH853660 \\
\hline Stagonosporopsis crystalliniformis & CBS $713.85^{\mathrm{T}}$ & GU238178 & GU237903 & KT389675 & GU237683 \\
\hline Stagonosporopsis cucurbitacearum & CBS 133.96 & GU238181 & GU237780 & KT389676 & GU237686 \\
\hline Stagonosporopsis dennisii & CBS $631.68^{\mathrm{T}}$ & GU238182 & GU237899 & KT389677 & GU237687 \\
\hline Stagonosporopsis dorenboschii & CBS $426.90^{\mathrm{T}}$ & GU238185 & GU237862 & KT389678 & GU237690 \\
\hline Stagonosporopsis helianthi & CBS $200.87^{\mathrm{T}}$ & KT389761 & KT389545 & KT389683 & KT389848 \\
\hline Stagonosporopsis heliopsidis & CBS 109182 & GU238186 & GU237747 & KT389679 & GU237691 \\
\hline Stagonosporopsis hortensis & CBS 104.42 & GU238198 & GU237730 & KT389680 & GU237703 \\
\hline Stagonosporopsis hortensis & CBS 572.85 & GU238199 & GU237893 & KT389681 & GU237704 \\
\hline Stagonosporopsis ligulicola var. ligulicola & CBS 500.63 & GU238190 & GU237871 & - & GU237695 \\
\hline
\end{tabular}


Table 4 Continued.

\begin{tabular}{|c|c|c|c|c|c|}
\hline \multirow{2}{*}{ Species $^{1}$} & \multirow{2}{*}{$\begin{array}{l}\begin{array}{l}\text { Culture } \\
\text { number }\end{array} \\
\end{array}$} & \multicolumn{4}{|c|}{ GenBank Accession Numbers $^{3}$} \\
\hline & & LSU & ITS & TUB & RPB2 \\
\hline Stagonosporopsis ligulicola var. ligulicola & CBS 137.96 & GU238191 & GU237783 & - & GU237696 \\
\hline Stagonosporopsis ligulicola var. inoxydabilis & CBS $425.90^{\mathrm{T}}$ & GU238188 & GU237861 & KT389682 & GU237693 \\
\hline Stagonosporopsis ligulicola var. inoxydabilis & PD $85 / 259$ & GU238189 & GU237920 & GU237694 & \\
\hline Stagonosporopsis loticola & CBS $562.81^{\mathrm{T}}$ & GU238192 & GU237890 & KT389684 & GU237697 \\
\hline Stagonosporopsis lupini & CBS $101494^{\mathrm{T}}$ & GU238194 & GU237724 & KT389685 & GU237699 \\
\hline Stagonosporopsis oculo-hominis & CBS $634.92^{\mathrm{T}}$ & GU238196 & GU237901 & KT389686 & GU237701 \\
\hline Stagonosporopsis papillata & $\mathrm{LC} 8169^{\mathrm{T}}$ & KY742279 & KY742125 & KY742191 & KY742367 \\
\hline Stagonosporopsis papillata & LC 8170 & KY742280 & KY742126 & KY742192 & KY742368 \\
\hline Stagonosporopsis papillata & LC 8171 & KY742281 & KY742127 & KY742193 & KY742369 \\
\hline Stagonosporopsis rudbeckiae & CBS 109180 & GU238197 & GU237745 & - & GU237702 \\
\hline Stagonosporopsis tanaceti & CBS $131484^{\mathrm{T}}$ & JQ897461 & NR_111724 & - & JQ897496 \\
\hline Stagonosporopsis trachelii & CBS 379.91 & GU238173 & GU237850 & KT389687 & GU237678 \\
\hline Stagonosporopsis trachelii & CBS 384.68 & GU238174 & GU237856 & - & GU237679 \\
\hline Stagonosporopsis valerianellae & CBS 273.92 & GU238200 & GU237819 & - & GU237705 \\
\hline Stagonosporopsis valerianellae & CBS $329.67^{\mathrm{T}}$ & GU238201 & GU237832 & - & GU237706 \\
\hline
\end{tabular}

${ }^{1}$ ATCC: American Type Culture Collection, USA; BRIP: Plant Pathology Herbarium, Department of Employment, Economic, Development and Innovation, Queensland, Australia; CBS: Westerdijk Fungal Biodiversity Institute, Utrecht, The Netherlands; CGMCC: China General Microbiological Culture Collection, Beijing, China; JZB: Beijing Academy of Agriculture and Forestry Sciences culture collection, Beijing, China; LC: Lei Cai's personal collection deposited in laboratory, housed at CAS, China; MFLUCC: Mae Fah Luang University Culture Collection, Chiang Rai, Thailand; PD: Plant Protection Service, Wageningen, the Netherlands; UTHSC, Fungus Testing Laboratory at the University of Texas Health Science Center, San Antonio, Texas, USA.

${ }^{2}$ Ex-type, neo-type and epi-type cultures are marked with superscript ${ }^{\mathrm{T}}$ and newly generated sequences are shown in bold face.

${ }^{3}$ ITS: internal transcribed spacer regions $1 \& 2$ including 5.8S nrDNA gene; LSU: 28S large subunit of the nrRNA gene; RPB2: RNA polymerase II second subunit; TUB: ß-tubulin.

\section{Results}

\section{Disease symptoms identified in the field}

During this study, symptomatic cherry leaves were observed in the fields at Academy of Forestry and Pomology Sciences, Beijing (Fig. 1). Initially, small, dark red or purple pinpoint lesions appear on the top leaf surface of infected leaves in early summer. With time these lesions enlarge to 
form red-brown, 1-3 mm circular lesions with evenly curved and smooth margins. In some leaves, these enlarged pinpoint spots combined together to form larger dead patches. Following 7-10 days of initial symptoms, necrotic tissues of the leaf spots drop out causing shot holes. Heavily infected leaves turn light green and yellow areas resulted from leaf chlorosis form around the spots. One month after the initial infection, leaves die off and fall from the tree leading to premature tree defoliation. Disease severity of the plants in the field is very high. Almost all of the trees were infected and most of the trees show heavy infection.

\section{Fungal isolation}

A total of 67 isolates were obtained from leaf spot tissues of approximately 60 diseased leaves collected from Prunus avium at Beijing Academy of Forestry and Pomology Sciences, Beijing, China. Among these, the majority were Alternaria species (58 isolates) and the rest divided among Colletotrichum (3 isolates) and didymellaceous taxa (6 isolates).

\section{Multi-locus phylogenetic analyses}

Phylogenetic analyses were conducted separately for Alternaria species, Colletotrichum species and for the species in Didymellaceae. The first phylogenetic tree focusses on the Alternaria section Alternaria, the second one was for Colletotrichum species and the last one was produced to estimate the phylogenies of Didymellaceae species. As described in Jeewon \& Hyde (2016), recommendations for base pair differences among the species were followed when introducing new species.
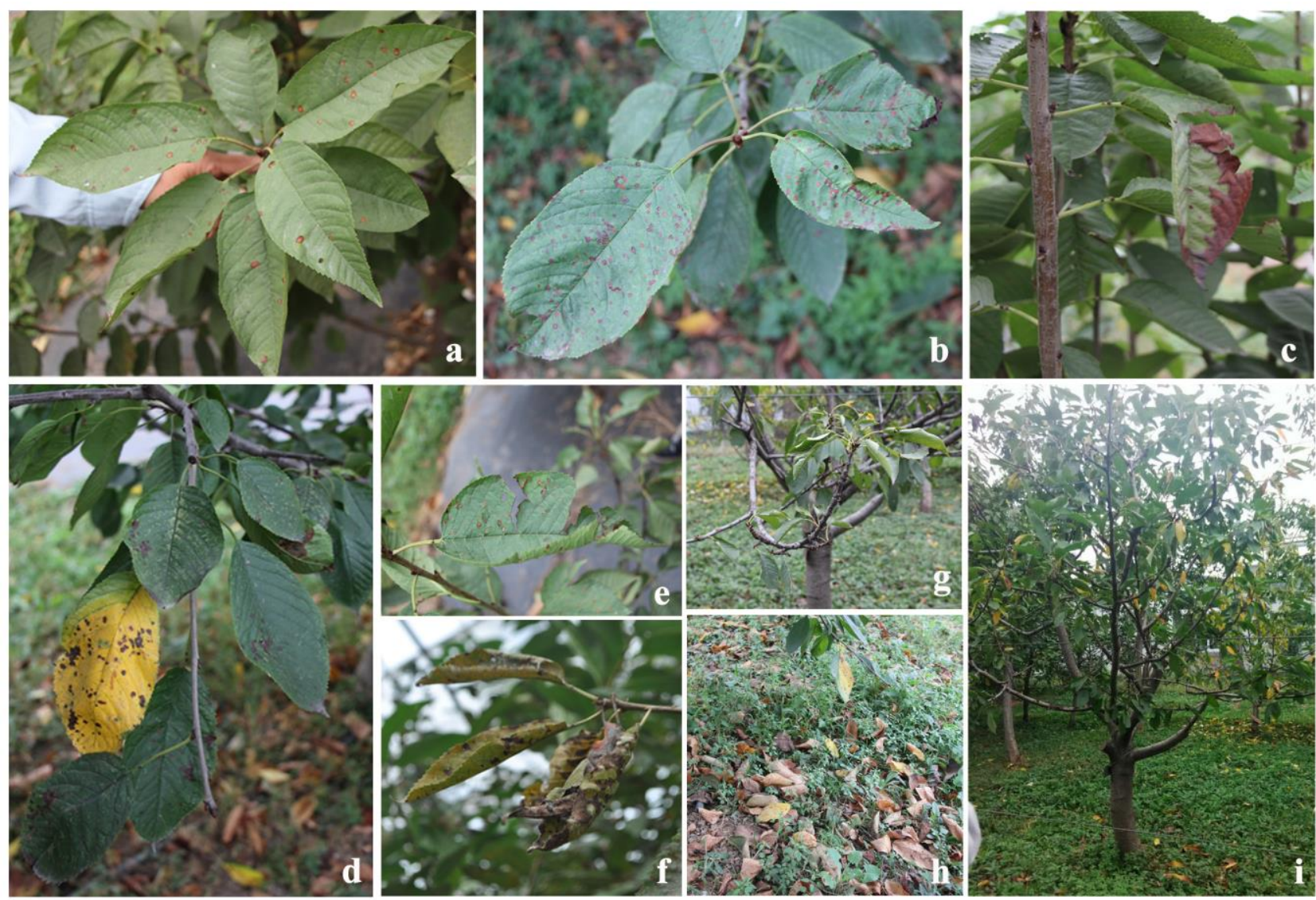

Figure 1 - Disease symptoms of Cherry leaf spot on Prunus avium in the field. a Development of numerous specks on leaves. b Small, dark red or purple pinpoint lesions on severely infected leaves. c Coalescing and formation of irregular necrotic patches. d Formation of chlorotic areas. e Formation of shot holes. f Curling of chlorotic and a-chlorotic leaves. g Premature defoliation. $h$ infected leaves on the ground. i Heavily infected tree. 
The phylogeny of genus Alternaria was defined by 76 strains of Alternaria species. In the phylogenetic analyses, 14 internal clades (herein called sections) occur consistently in the individual and combined phylogenies. These include sections Alternaria, Alternantherae, Brassicicola, Cheiranthus, Dianthicola, Eureka, Gypsophilae, Japonicae, Panax, Pseudoulocladium, Porri, Radicina, Sonchi, Teretispora and Ulocladioides. Several gene combinations were tested to obtain the best resolution for the identification of Alternaria

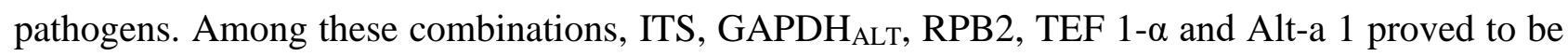
the best combination. All the isolates from the current study clustered in a subclade within section Alternaria (Supplementary Fig. 1). Therefore, separate phylogenetic analyses were conducted for Alternaria section Alternaria. Alternaria section Alternaria combined dataset consists of 42 sequences representing 15 taxa with Alternaria alternantherae (CBS 124392) of section Alternantherae as the outgroup. Multi-gene phylogenetic trees with similar topologies were generated from MP, ML and Bayesian analyses. The parsimony analysis comprised 2681 total characters including gaps. The concatenated alignment contained 179 parsimony informative characters, 184 variable and parsimony-uninformative characters, and 2318 constant characters. The first of 1000 equally parsimonious trees is shown in Fig. 2, which enabled the identification of the isolates to species level, with a better resolution than the single-gene analyses ( $\mathrm{TL}=477$, $\mathrm{CI}=0.830, \mathrm{RI}=0.877, \mathrm{RC}=0.728, \mathrm{HI}=0.170$ ). Maximum likelihood matrix had 309 distinct alignment patterns, with $10.48 \%$ of undetermined characters or gaps. Estimated base frequencies were as follows; $\mathrm{A}=0.239957, \mathrm{C}=0.281729, \mathrm{G}=0.243707, \mathrm{~T}=0.234608$; substitution rates $\mathrm{AC}$ $=1.097417, \mathrm{AG}=2.879682, \mathrm{AT}=0.925667, \mathrm{CG}=0.528440, \mathrm{CT}=5.893520, \mathrm{GT}=1.000000$; gamma distribution shape parameter $\alpha=0.107840$. All our isolates divided among three species. Combined phylogenetic analyses provided good evidence that most of the isolates in the current study belong to a new species, which we introduce in this study as Alternaria prunicola, with high bootstrap values and high Bayesian posterior probabilities (MP: 81\%, ML: 85\%, BPP: 1.00). Many of the isolates of the current study were clustered together with Alternaria prunicola. Hence, it was considered as the main pathogen of Cherry leaf spot disease in Beijing, China. In addition, another new species Alternaria pseudoeichhorniae was identified with high bootstrap values and high Bayesian posterior probabilities (MP: 100\%, ML: 95\%, BPP: 1.00). Several Alternaria isolates were clustered with the ex-type of previously identified pathogen Alternaria alternata (CBS 916.96). In the phylogenetic analyses, Alternaria alternata isolates clustered into two sub clades. This is similar to the study conducted by Woudenberg et al. (2015) where they clustered into many sub clades. During this study, 35 morphospecies were synonymized under A. alternata due to their inability to be reliably distinguished in the multi-gene phylogeny. In the current study, we have selected several $A$. alternata isolates representing these clades. Similarly, we have also observed several sub clades in our analyses for the synonymized A. alternata isolates.

For the identification of Colletotrichum isolates, a phylogenetic tree was constructed using 63 representative Colletotrichum isolates including the isolates from the current study. Based on NCBI GenBank BLASTn search, all Colletotrichum isolates of the current study belong to $C$. gloeosporioides species complex. Concatenated analyses of ITS, GAPDH, CHS-1, ACT and TUB2 were performed for the $C$. gloeosporioides species complex. The dataset consists of 62 sequences representing 42 taxa with Colletotrichum boninense (MAFF 305972) representing the outgroup. The trees generated from the Bayesian and ML analyses share a similar topology from that of the MP analysis (Fig. 3). The parsimony analysis comprised 1941 total characters including gaps. The concatenated alignment consists of 361 parsimony informative characters, 250 variable and parsimony-uninformative characters and 1330 constant characters. The first of 1000 equally parsimonious trees is shown in Fig. 3, which enabled the identification of the isolates to the species level, with a better resolution than the single-gene analyses $(\mathrm{TL}=1077, \mathrm{CI}=0.703, \mathrm{RI}=0.852$, $\mathrm{RC}=0.599, \mathrm{HI}=0.297$ ). Maximum Likelihood alignment matrix had 694 distinct alignment patterns, with $13.50 \%$ of undetermined characters or gaps. Estimated base frequencies were as follows; $\mathrm{A}=$ $0.229422, \mathrm{C}=0.297559, \mathrm{G}=0.244522, \mathrm{~T}=0.228496$; substitution rates $\mathrm{AC}=1.040111, \mathrm{AG}=$ 2.595057, $\mathrm{AT}=0.828774, \mathrm{CG}=0.691054, \mathrm{CT}=4.320904, \mathrm{GT}=1.000000$; gamma distribution 
shape parameter $\alpha=0.388664$. In Fig. 3, two of our C. aenigma isolates (MFLUCC 18-1603 and MFLUCC 18-1604) clade together strongly with the ex-type strain of $C$. aenigma (ICMP 18608) and another $C$. pseudotheobromicola isolate (MFLUCC 18-1602) clade together with the ex-type of $C$. theobromicola (ICMP 18649).

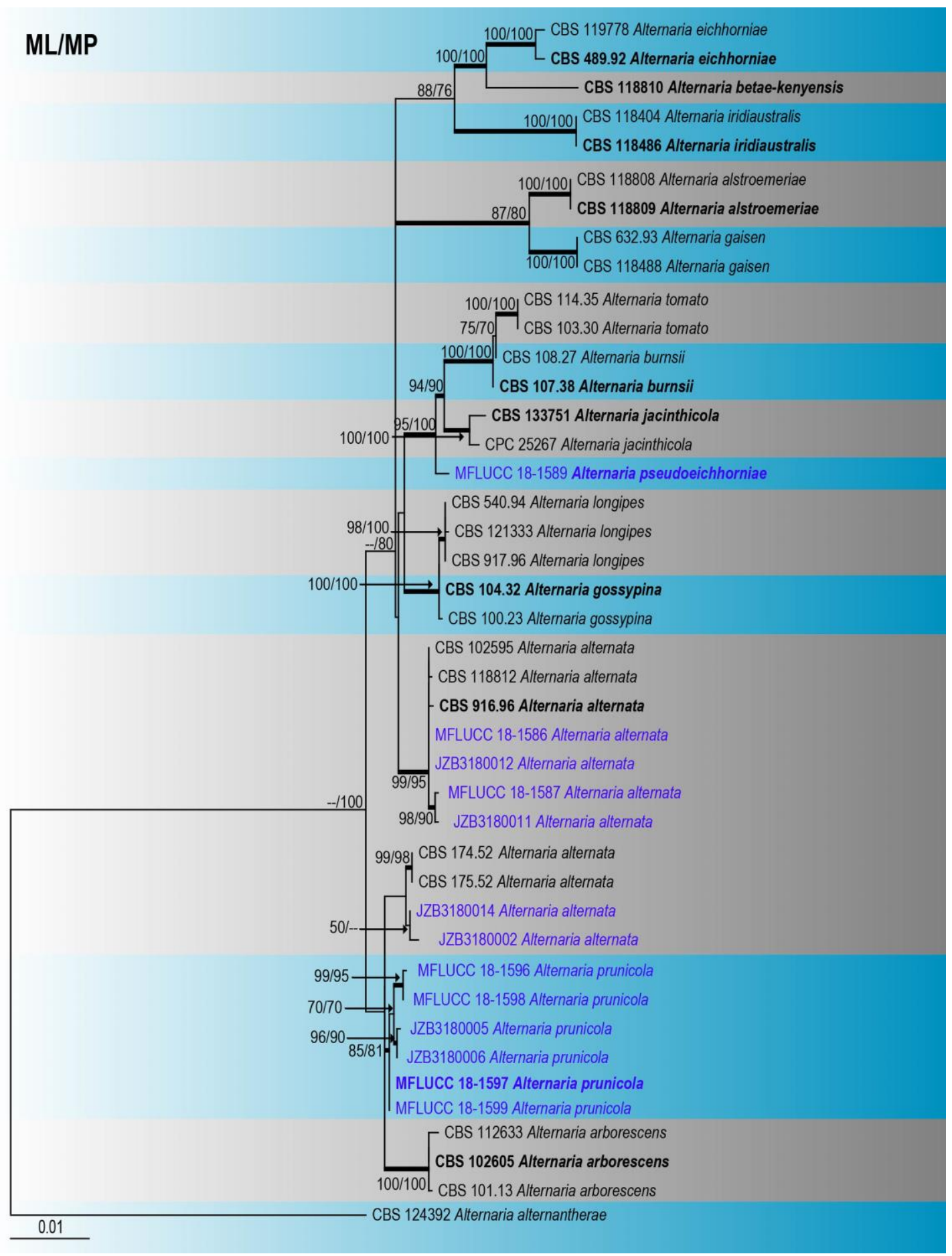

Figure 2 - Phylogenetic tree generated by maximum likelihood analysis of combined ITS, GADPH, RPB2, TEF 1- $\alpha$ and Alt-a 1 sequence data of species belonging to Alternaria alternata section Alternaria. The tree was rooted with Alternaria alternantherae (CBS 124392). Maximum parsimony and RAxML bootstrap support values $\geq 50 \%$ (BT) are shown respectively near the nodes. Bayesian posterior probabilities $\geq 0.95$ (PP) indicated as thickened black branches. The scale bar indicates 0.01 changes. The ex-type strains are in bold and isolates from the current study are in blue. 


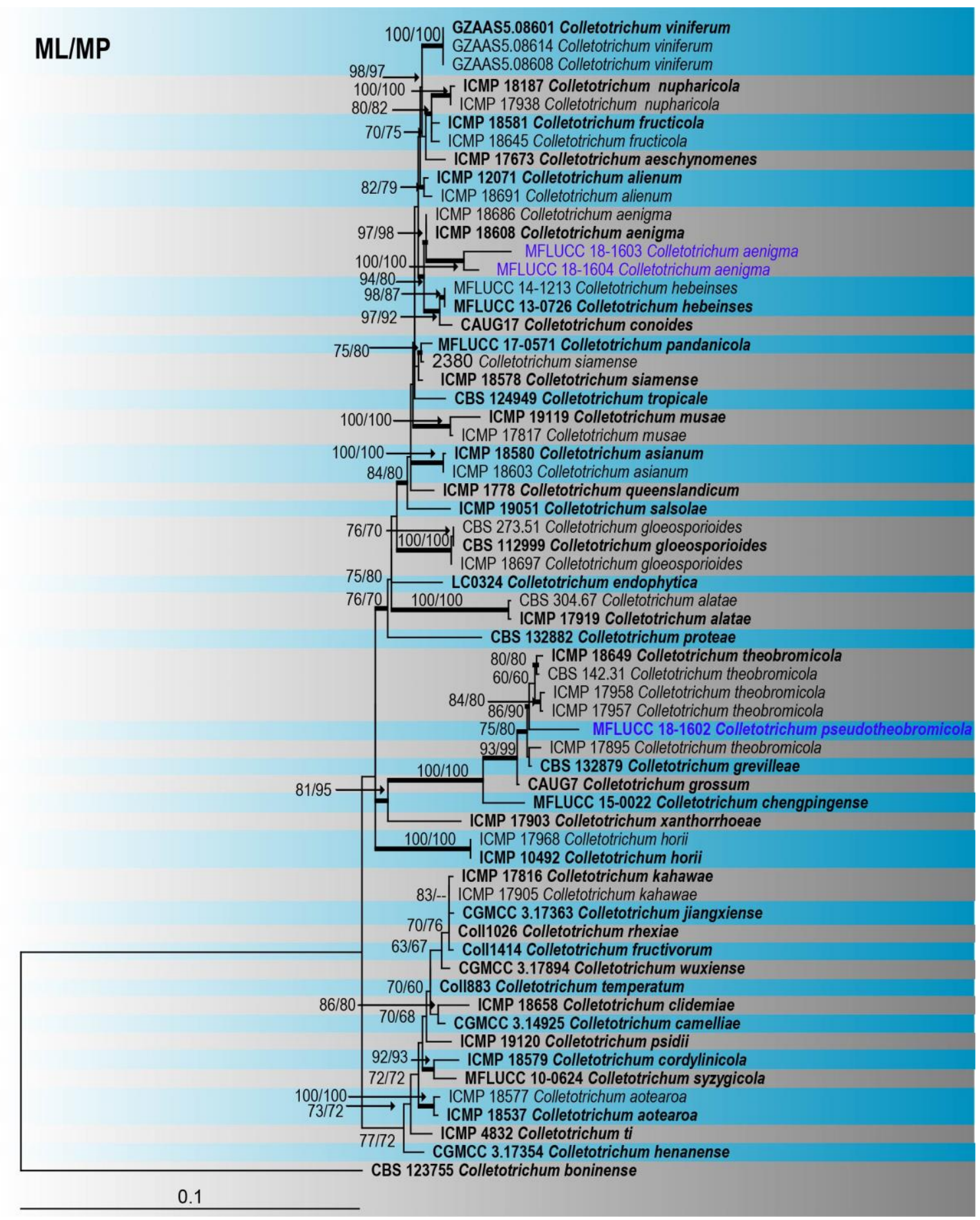

Figure 3 - Phylogenetic tree generated by maximum parsimony analysis of combined ITS, GAPDH, CHS-1, ACT and TUB2 sequence data of Colletotrichum gloeosporioides species complex. The tree was rooted with Colletotrichum boninense (CBS 123755). Maximum parsimony and RAxML bootstrap support values $\geq 50 \%$ (BT) are shown respectively near the nodes. Bayesian posterior probabilities $\geq 0.95$ (PP) indicated as thickened black branches. The scale bar indicates 10 changes. The ex-type strains are in bold and new isolates in blue.

Identification of Didymellaceae species was conducted using a concatenated multigene phylogenetic analysis of LSU, ITS, RPB2, and TUB2 gene regions. The Didymellaceae alignment included 113 strains, representing three genera with Neocucubitaria aquatica (CBS 297.74) as the outgroup, and consisted of 2328 characters forming 703 unique alignment patterns. Similar topology multigene phylogenetic trees were generated from the Bayesian, ML and MP analyses (Fig. 4). The parsimony analysis comprised 2328 total characters including gaps. The concatenated 
alignment consists of 452 parsimony informative characters, 274 variable and parsimonyuninformative characters, and 1602 constant characters. The first of 1000 equally parsimonious trees is shown in Fig. 4, which enabled the identification of the isolates to species level (TL $=2601$, $\mathrm{CI}=0.392, \mathrm{RI}=0.800, \mathrm{RC}=0.314, \mathrm{HI}=0.608$ ). Maximum likelihood alignment consisted of $9.08 \%$ of undetermined characters or gaps. Estimated base frequencies were as follows; $\mathrm{A}=0.238604, \mathrm{C}=$ $0.244685, \mathrm{G}=0.274276, \mathrm{~T}=0.242435$; substitution rates $\mathrm{AC}=1.272245, \mathrm{AG}=3.581243, \mathrm{AT}=$ $1.481142, \mathrm{CG}=0.853475, \mathrm{CT}=8.586284, \mathrm{GT}=1.000000$; gamma distribution shape parameter $\alpha$ $=0.175954$. In the current tree (Fig. 4), one of our Nothophoma quercina isolate (MFLUCC 181588) clustered together with the reference strain of $N$. quercina (CBS 633.92) and another four isolates, which were introduced as new species, N. pruni (JZB380015, MFLUCC 18-1601, JZB380017 and MFLUCC 18-1600) phylogenetically distinct from $N$. quercina reference strain (CBS 633.92). Stagonosporopsis citrulli isolate (MFLUCC 18-1595) clustered with the ex-type isolates of S. citrulli (ATCC TSD-2) and an Epicoccum pseudokeratinophilum isolate (MFLUCC 18-1593) clustered with the ex-type isolate of E. keratinophilum (UTHSCDI 16-271) as new species.

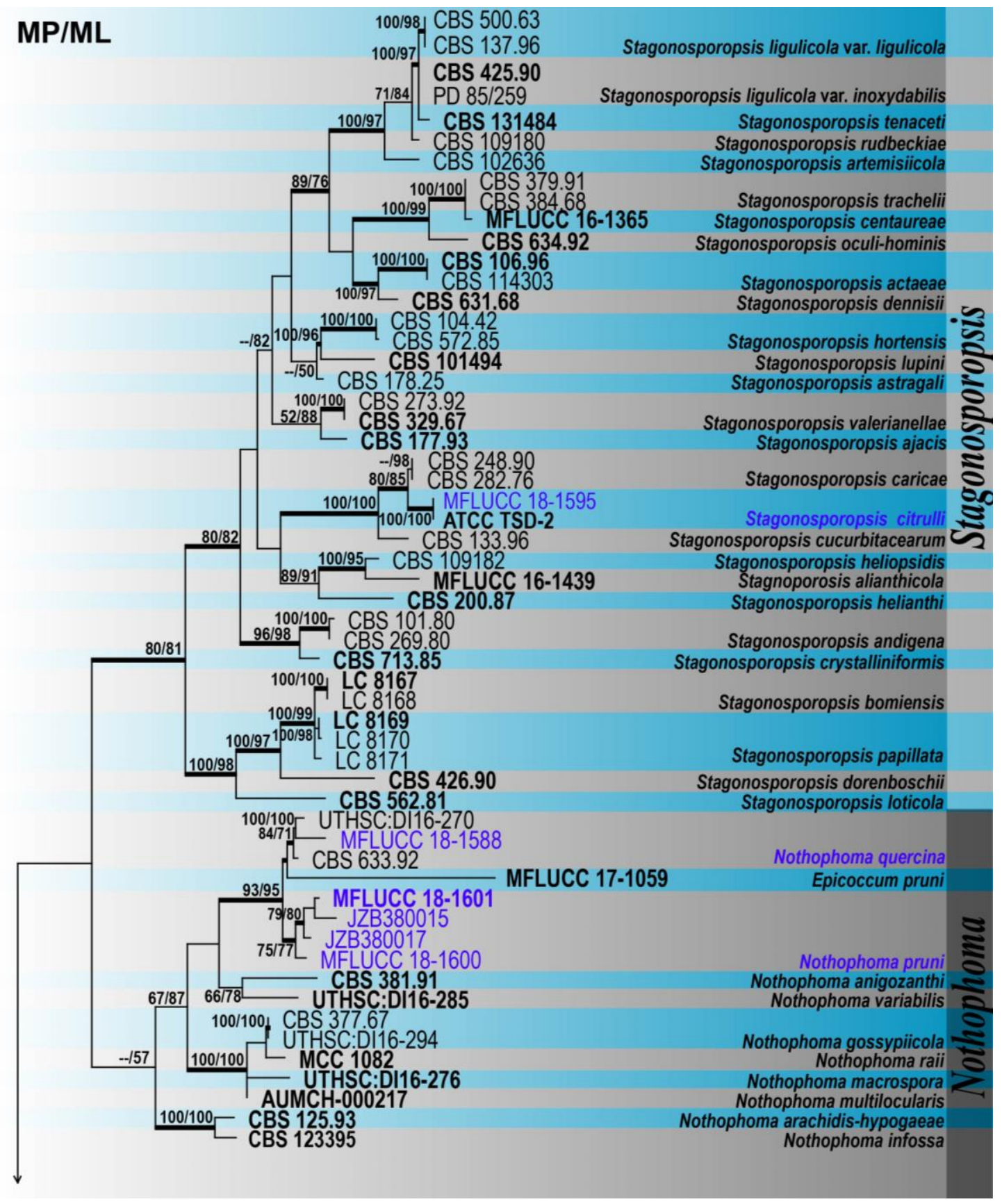




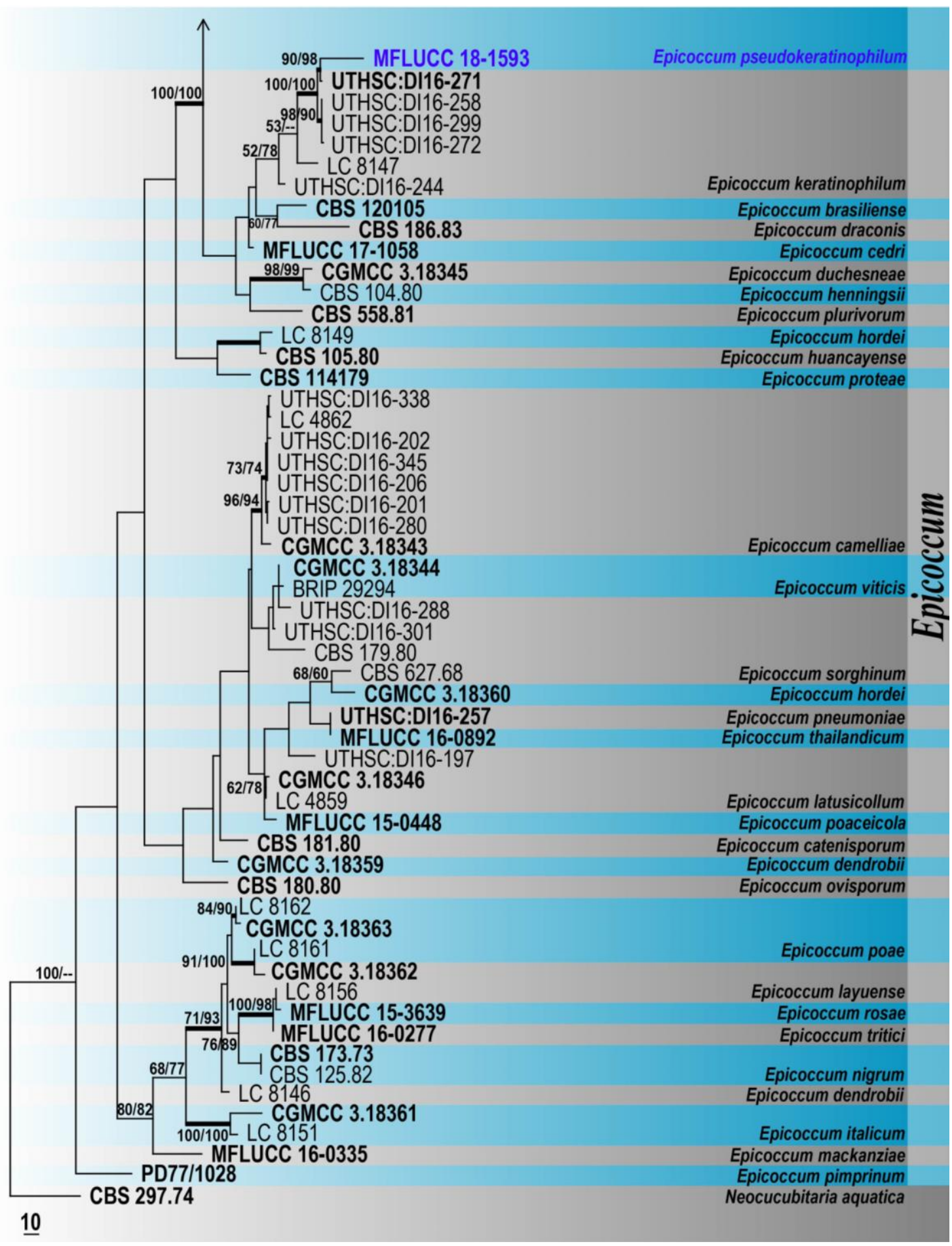

Figure 4 - Phylogenetic tree generated by maximum likelihood analysis of combined LSU, ITS, RPB2 and TUB2 sequence data of Didymellaceae species. The tree was rooted with Neocucubitaria aquatica (CBS 297.74). RAxML and Maximum parsimony bootstrap support values $\geq 50 \%$ (BT) are shown respectively near the nodes. Bayesian posterior probabilities $\geq 0.95$ (PP) indicated as thickened black branches. The scale bar indicates 0.1 changes. The ex-type strains are in bold and new isolates in blue.

\section{Pathogenicity assay}

An isolate of Alternaria alternata (MFLUCC 18-1587), two isolates of A. prunicola (MFLUCC 18-1597, MFLUCC 18-1599), two isolates of Colletotrichum aenigma (MFLUCC 181603, MFLUCC 18-1604), an isolate of C. pseudotheobromicola (MFLUCC 18-1602), an isolate of Epicoccum pseudokeratinophilum (MFLUCC 18-1593), an isolate of Stagonosporopsis citrulli 
(MFLUCC 18-1595) and an isolate of Nothophoma pruni (MFLUCC 18-1600) were subjected to detached leaf inoculation assay on three cultivars of Prunus avium.

Initially, pinpoint necrotic spots were formed near the inoculated area on leaves. Three days after inoculation, evenly round lesions surrounded by achlorotic margin were recorded on wounded inoculated leaves. These symptoms were similar to the characteristic lesions of cherry leaf spot that were observed in the field. After five days, lesions expanded and coalesced together to form larger necrotic areas. Colletotrichum species, being the highly pathogenic taxa, formed larger, dark brown necrotic areas. The major pathogen, A. prunicola formed identical necrotic areas for all of its isolates. Compared to these two genera, Didymellaceae species formed insignificant or no necrotic areas on the wounded leaves. No symptoms were observed on non-wounded inoculated leaves or on wounded and non-wounded leaves maintained as controls. Re-isolation from lesions confirmed the inoculated fungus based on cultural and morphological characters such as colony characters and conidial characters.

According to one-way ANOVA analysis, significantly different lesion areas resulted from different isolates $(\mathrm{F} 8=6.57, \mathrm{p}=0.000)$. Mean difference in the lesion areas were significantly highest between Epicoccum pseudokeratinophilum (lowest lesion area) and Colletotrichum pseudotheobromae (highest lesion area). Furthermore, significantly different lesion areas resulted in different cherry cultivars $(\mathrm{F} 8=6.42, \mathrm{p}=0.002)$. Mean lesion area of Prunus avium cv. 'Summit' was significantly different from mean lesion areas of Prunus avium cv. 'Tieton' and 'Sunburst'. Lesion areas formed by all of the isolates on Prunus avium cv. 'Summit' were larger, while smallest lesion areas were formed on Prunus avium cv. 'Sunburst'. Therefore, based on the analysis Prunus avium cv. 'Summit' was highly susceptible to cherry leaf spot disease whereas Prunus avium cv. 'Sunburst' showed the highest resistance to the disease. Two-way ANOVA analysis for lesion area showed that there was a significant interaction between the pathogen and cherry cultivar for forming lesion areas (Table 5) and the interaction plot for lesion area demonstrated these relationships (Figs 5, 6).

Table 5 Two-way analysis of variance for lesion area $v s$. isolate and variety

\begin{tabular}{llllll}
\hline Source & $\mathbf{D F}^{\mathbf{1}}$ & $\mathbf{S S}^{\mathbf{1}}$ & $\mathbf{M S}^{\mathbf{1}}$ & $\mathbf{F}^{\mathbf{1}}$ & $\mathbf{P}^{\mathbf{1}}$ \\
\hline Isolate & 8 & 18.2010 & 2.27513 & 8.81 & 0.000 \\
Variety & 2 & 5.4798 & 2.73988 & 10.61 & 0.000 \\
Interaction (isolate $\times$ interaction) & 16 & 10.2596 & 0.64123 & 2.48 & 0.003 \\
Error & 108 & 27.8824 & 0.25817 & & \\
Total & 134 & 61.8229 & & & \\
\hline
\end{tabular}

${ }^{1}$ DF: Degrees of Freedom; SS: Sums of Squares; MS: Mean Squares; F: F-value; P: P-value

\section{Taxonomy}

Pleosporaceae Nitschke, Verh. naturh. Ver. preuss. Rheinl. 26: 74 (1869)

Alternaria Nees, Syst. Pilze (Würzburg): 72. 1816 [1816-1817]

Alternaria, introduced by Nees von Esenbeck (1816), is an ubiquitous genus treated under Pleosporaceae, Pleosporales, Dothideomycetes. The genus includes saprobes, endophytes and plant pathogens associated with a wide variety of substrates (Woudenberg et al. 2013). The genus has been subjected to several major revisions during the last few years (Woudenberg et al. 2013, Ariyawansa et al. 2015).

Alternaria alternata (Fr.) Keissl., Beih. Bot. Centralbl., Abt. 2 29: 434 (1912) 


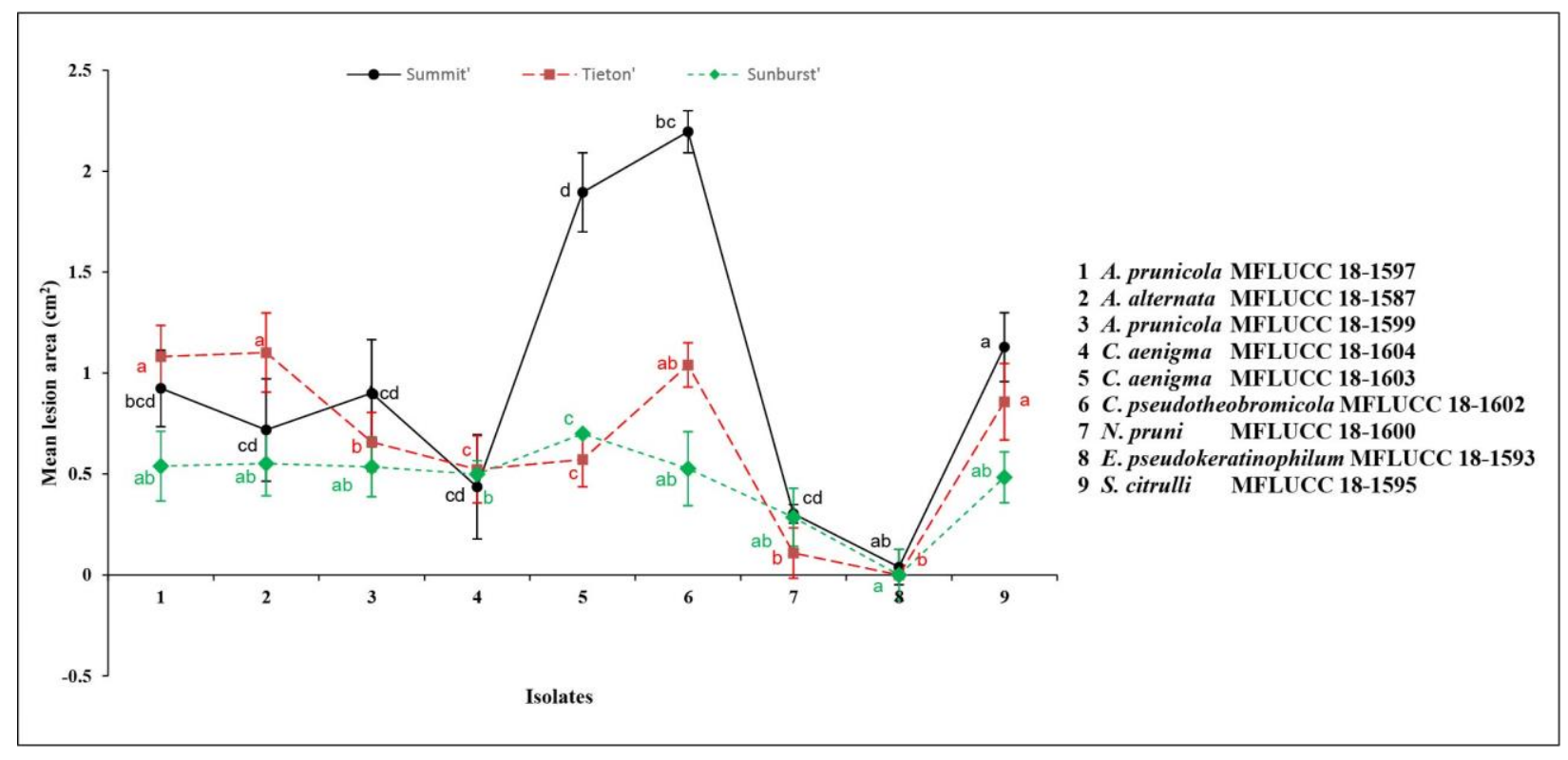

Figure 5 - Variations in the virulence of lesion areas of pathogenic isolates on cherry leaves resulting from the pathogenicity test three days post inoculation. Virulence of isolates depends on both the isolates as well as on the cherry cultivar. Characters indicate the significant differences for lesion areas. Isolates that do not share the same letter are significantly different.

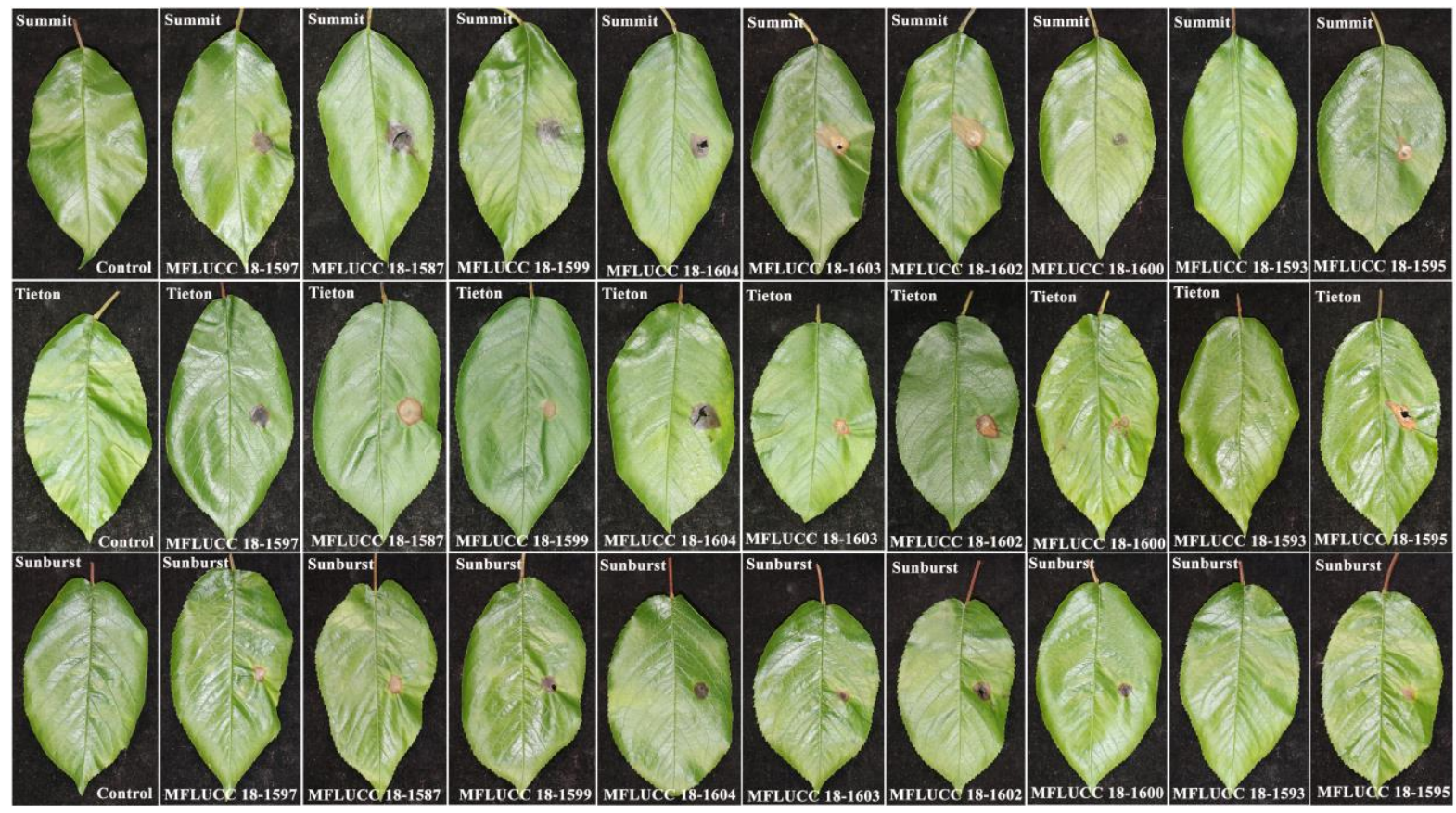

Figure 6 - Detached leaf pathogenicity assay for the fungal isolates on P. avium cvs. 'Summit', 'Tieton' and 'Sunburst' three days post inoculation. Symptoms on leaves symmetrically inoculated with a conidial suspension of the isolated pathogen, right: non-wounded inoculation; left: wounded inoculation. Top right corner of each figure indicate the $P$. avium cultivar name and the bottom left indicate the inoculated fungal isolate number. The species names are as follows: MFLUCC 181587 = Alternaria alternata, MFLUCC 18-1597, MFLUCC 18-1599 = Alternaria prunicola, MFLUCC 18-1603, MFLUCC 18-1604 = Colletotrichum aenigma, MFLUCC 18-1602 = Colletotrichum pseudotheobromicola, MFLUCC 18-1593 = Epicoccum pseudokeratinophilum, MFLUCC 18-1595 = Stagonosporopsis citrulli and MFLUCC 18-1600 = Nothophoma pruni . Sterilized water was used as the control. The highest level of virulence was exhibited by Colletotrichum species. All Didymellaceae species induced less or no lesions on the cherry leaves. 
Pathogenic on diseased leaves of Prunus avium. Sexual morph: Undetermined. Asexual morph: Hyphae superficial or submerged, subhyaline, branched, smooth to verruculose, septate, 2$3 \mu \mathrm{m}$ wide. Conidiophores $23-40 \times 3-5 \mu \mathrm{m}(\overline{\mathrm{x}}=27 \times 3.5 \mu \mathrm{m}, \mathrm{n}=20)$, solitary, simple or branched, brown, multi-septate, with a single terminal conidiogenous loci. Conidia $20-40 \times 9-15 \mu \mathrm{m}(\overline{\mathrm{x}}=29$ $\times 10.2 \mu \mathrm{m}, \mathrm{n}=40$ ), solitary or in branched chains of 20 or more, first $1-2$ conidia in each chain longer than others, straight, ellipsoidal or ovoid, pale to dark brown to olivaceous green, with smooth outer wall, some muriform, usually with 1-6 transverse septa and 0-3 longitudinal septa, rounded apex. Conidial beaks pale brown to subhyaline, not branched, 2-6 $\times 3-4 \mu \mathrm{m}$.

Culture characteristics - Colonies on PCA attaining $80 \mathrm{~mm}$ diam. after 5 days at $25{ }^{\circ} \mathrm{C}$, circular, entire-edged, flat, floccose to woolly, surface pale olivaceous grey near the margin changing to dull green in the centre and reverse olivaceous black in the centre and pale olivaceous grey near the margin.

Material examined - CHINA. Beijing, on leaf spots of Prunus avium L. (Rosaceae), 28 September 2017, K.W.T. Chethana (MFLU 18-2659) - living culture, MFLUCC 18-1586, KUMCC 18-0394; ibid. (MFLU 18-2660) - living culture, MFLUCC 18-1587, KUMCC 180395.

Additional material examined - CHINA. Beijing, on leaf spots of Prunus avium L. (Rosaceae), 28 September 2017, K.W.T. Chethana (JZB-H 3180012) - living culture, JZB3180012; ibid. (JZB-H 3180011) - living culture, JZB3180011; ibid. (JZB-H 3180014) - living culture, JZB3180014; ibid. (JZB-H 3180002) - living culture, JZB3180002.

Notes - Alternaria alternata is a common pathogen of many hosts and mostly found as saprobes (Thomidis \& Tsipouridis 2006, Hyde et al. 2009, Jayawardena et al. 2016). There is a report on A. alternata causing leaf spot on cherry in Greece (Thomidis \& Tsipouridis 2006). Based on our phylogenetic analysis of combined ITS, GAPDH, RPB 2 , TEF 1- $\alpha$ and Alt-a sequence data of Alternaria species (Fig. 2), some of our isolates (MFLUCC 18-1586, JZB3180012,) clustered together with the ex-type strain of A. alternata (CBS 916.96) with high bootstrap and Bayesian probabilities (99\% MP, 95\% ML and 1.00 PP), while others (JZB3180011, JZB3180014, JZB3180002 and MFLUCC 18-1587) are phylogenetically distant. As discussed under multi-gene phylogenies, this phylogenetically distant group is due to the inclusion of different morphospecies used in the study which are currently synonymized under A. alternata. Comparisons of base pair differences for all the genes between our strain (MFLUCC 18-1586) and ex-type strain of $A$. alternata (CBS 916.96) reveal identical or less than $1 \%$ base pair differences. When our strain was compared with the type specimen of A. alternata (CBS 916.96), it showed similar morphology (Ariyawansa et al. 2015, Woudenberg et al. 2015).

Alternaria prunicola Chethana, Yan, Li \& K.D. Hyde, sp. nov.

Fig. 8

MycoBank number: MB828515; Facesoffungi number: FoF04913

Etymology - The specific epithet prunicola was given after the host genus.

Pathogenic on diseased leaves of Prunus avium. Sexual morph: not observed. Asexual morph: Hyphae subhyaline to pale olivaceous, branched, smooth, septate, 3-4 $\mu \mathrm{m}$ wide. Conidiophores 13-40 $\times 2.5-5 \mu \mathrm{m}(\overline{\mathrm{x}}=26.5 \times 3.8 \mu \mathrm{m}, \mathrm{n}=20)$, solitary, simple, straight or flexuous, dark brown, multi-septate, with a single or two terminal conidiogenous loci. Conidia $18-37.1 \times 6-$ $15 \mu \mathrm{m}(\overline{\mathrm{x}}=25.5 \times 8.8 \mu \mathrm{m}, \mathrm{n}=40)$, solitary or in branched chains of 4 or more, straight, clavate to elongated clavate, olivaceous to light brown, with smooth outer wall, some muriform, usually with 3-4 transverse septa and 0-1 longitudinal septa, rarely have oblique septa which divide the septate cells into cuboid portions, often constricted at the primary septa, rounded apex, stalked or stalkless.

Culture characteristics - Colonies on PCA attaining $80 \mathrm{~mm}$ diam. after 5 days at $25{ }^{\circ} \mathrm{C}$, circular, entire-edged, effuse, floccose to woolly, surface pale olivaceous grey near the margin changing to dull green in the centre and reverse olivaceous black in the centre and pale olivaceous grey near the margin.

Material examined - CHINA. Beijing, on leaf spots of Prunus avium L. (Rosaceae), 28 September 2017, K.W.T. Chethana (MFLU 18-2661, holotype), ex-type culture, MFLUCC 18- 
1597; ibid. (KUMCC 18-0405, isotype); ibid. (MFLU 18-2662) - living culture, MFLUCC 181596, KUMCC 18-0404; CHINA. ibid. (MFLU 18-2663) - living culture, MFLUCC 18-1599, KUMCC 18-0407; ibid. (MFLU 18-2664) - living culture, MFLUCC 18-1598, KUMCC 180406.

Additional material examined - CHINA. Beijing, on leaf spots of Prunus avium L. (Rosaceae), 28 September 2017, K.W.T. Chethana (JZB-H 3180005) - living culture, JZB3180005; ibid. (JZB-H 3180006) - living culture, JZB3180006.
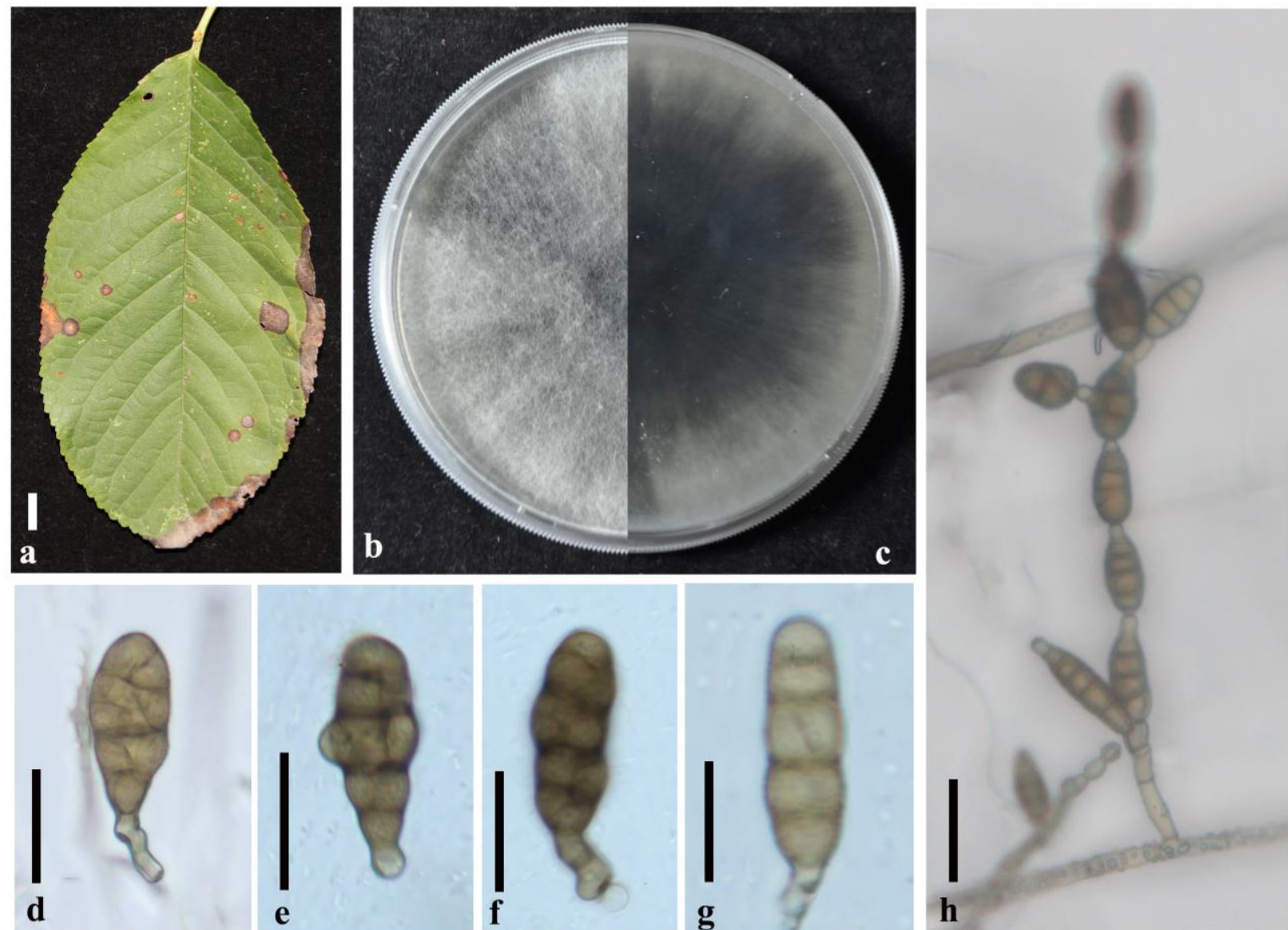

Figure 7 - Alternaria alternata (MFLUCC 18-1586) a Host surface from which the pathogen was isolated. b, c Upper-view (b) and the reverse view (c) of the colony on PCA. d-g Beaked or beakless different conidial morphologies. h Sporulation pattern of A. alternata. Scale bars: $\mathrm{a}=1$ $\mathrm{cm}, \mathrm{d}-\mathrm{g}=20 \mu \mathrm{m}, \mathrm{h}=50 \mu \mathrm{m}$.

Note - In our phylogenetic analysis of combined ITS, GAPDH, RPB 2 , TEF 1- $\alpha$ and Alt-a sequence data of Alternaria species (Fig. 2), all of the isolates belonging to Alternaria prunicola formed a subclade within section Alternaria. Alternaria prunicola was well-separated from other Alternaria species with a strong $82 \%$ ML, $81 \%$ MP bootstrap values and 1.00 posterior probability; its sister taxa A. alternata (CBS 919.96) and A. longipes (CBS 540.94) clustered separately from $A$. prunicola with $100 \% \mathrm{MP}, 100 \% \mathrm{ML}$ and 1.00 posterior probabilities. A comparison of the 509 nucleotides across the ITS $(+5.8$ S) gene region between A. prunicola (MFLUCC 18-1597) and its sister taxa A. alternata (CBS 919.96) and A. longipes (CBS 540.94) reveal 22.09\% and 22.28\% base pair differences respectively. In addition, we compared our new taxon with A. alternata (CBS 919.96) and A. longipes (CBS 540.94) for base pair differences in the protein coding genes and there are $2.86 \%$ and $3.43 \%$ base pair differences respectively across 524 nucleotides in GAPDH gene region; $1.66 \%$ and $8.73 \%$ base pair differences respectively across 1070 nucleotides in $\mathrm{RPB}_{2}$ gene region; $8.33 \%$ and $10.13 \%$ base pair differences respectively across 202 nucleotides in TEF 1- 
$\alpha$ gene region. Another Alternaria species, Alternaria pruni McAlpine had been isolated from Apricot leaves. However, it is morphologically different from our collection in having larger (52$64 \times 13-18 \mu \mathrm{m}$ ), 6-8 septate spores (McAlpine 1902). Due to the unavailability of DNA sequences, this was not included in the phylogenetic analysis. Our collection is distinct from A. alternata, another reported cherry leaf spot pathogen, in having clavate to elongated clavate, 3-4 transverse septate and $0-1$ longitudinal septate, smaller conidia $(18-37.1 \times 6-15 \mu \mathrm{m})$, in contrast to obclavate, obpyriform, ovoid or ellipsoidal, pale to mid golden brown, 8 transverse and usually several longitudinal or oblique septate, larger $(20-63 \times 9-18 \mu \mathrm{m})$ conidia of A. alternata (Ellis 1971).
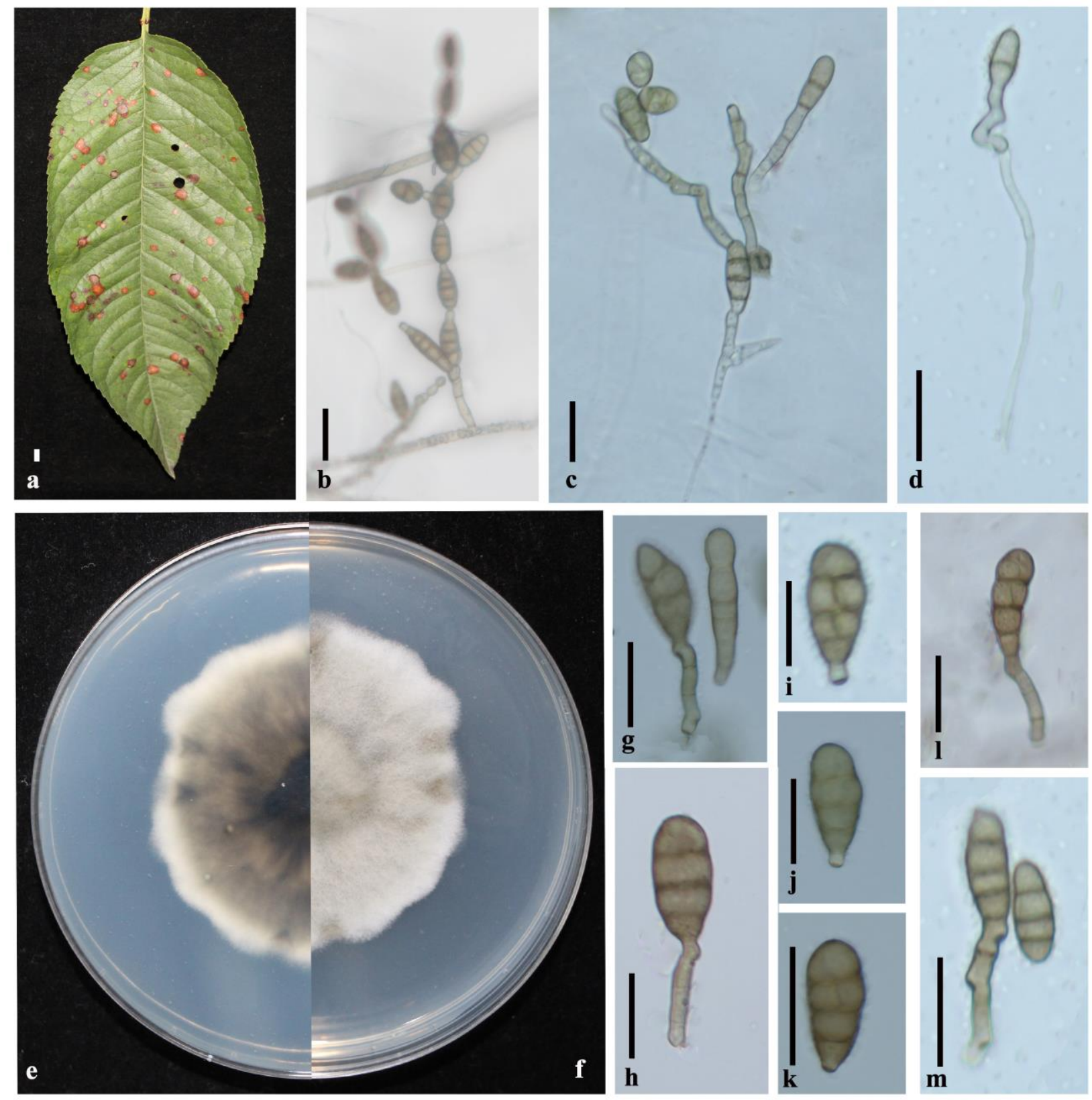

Figure 8 - Alternaria prunicola (MFLUCC 18-1597, holotype) a Host surface from which the pathogen was isolated. b Sporulation pattern of A. prunicola. c Conidiophores connected to conidia. $\mathrm{d}$ Germinating conidia. e, f Upper-view (e) and the reverse view (f) of the colony on PCA. $\mathrm{g}-\mathrm{m}$ Stalked or stalkless different conidial morphologies. Scale bars: $\mathrm{a}=3 \mathrm{~mm}, \mathrm{~b}-\mathrm{d}, \mathrm{g}-\mathrm{m}=20 \mu \mathrm{m}$.

Alternaria pseudoeichhorniae Chethana, Yan, Li \& K.D. Hyde, sp. nov.

Fig. 9 MycoBank number: MB828516; Facesoffungi number: FoF04912 
Etymology - The specific epithet pseudoeichhorniae was given after its close resemblance to Alternaria pseudoeichhorniae.

Pathogenic on diseased leaves of Prunus avium. Sexual morph: not observed. Asexual morph: Hyphae subhyaline to hyaline, branched, smooth, septate. Conidiophores 18-48.5 × 2.5-6 $\mu \mathrm{m}(\overline{\mathrm{x}}=29.1 \times 4.3 \mu \mathrm{m}, \mathrm{n}=10)$, solitary, simple, straight or flexuous, dark brown, multi-septate, with a single or up to three terminal conidiogenous loci. Conidia $16-30.2 \times 5-13 \mu \mathrm{m}(\overline{\mathrm{x}}=22.6 \times$ $9.8 \mu \mathrm{m}, \mathrm{n}=40$ ), solitary or in a chain of $2-4$ or more, straight, obpyriform to obclavate, light brown, with smooth outer wall, usually with an indistinct basal pore, muriform, with 2-3 transverse and 0-1 longitudinal septa, often constricted at the primary septa. Conidial beak absent or present as a short conical, narrowly tapered or almost cylindrical beak.
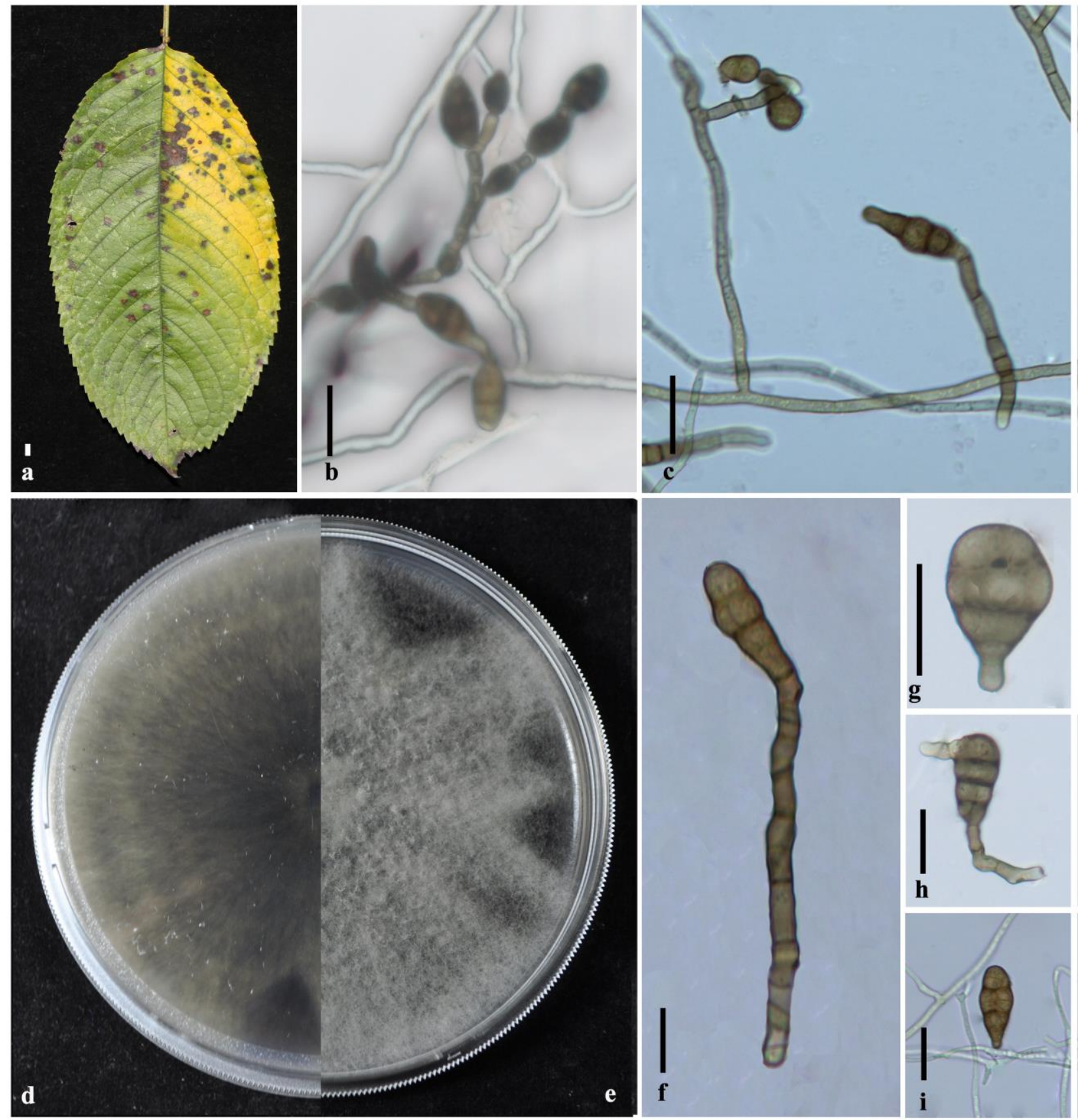

Figure 9 - Alternaria pseudoeichhorniae (MFLUCC 18-1589, holotype) a Host surface from which the pathogen was isolated. b Sporulation pattern of A. eichhorniae. c Conidiophore. $\mathrm{d}$, e Upper-view (e) and the reverse view (d) of the colony on PCA. f Beaked conidium. $\mathrm{g}-\mathrm{i}$ Germinating beakless conidia. Scale bars: $\mathrm{a}=3 \mathrm{~mm}, \mathrm{~b}, \mathrm{c}, \mathrm{f}-\mathrm{i}=20 \mu \mathrm{m}$. 
Culture characteristics - Colonies on PCA fast growing, circular, with velvety to cotton abundant greyish aerial mycelium, effuse at the edges, occasionally forming black patches towards the margin of the colonies, with conspicuous concentric zonations of growth, attaining a diameter of $8.5 \mathrm{~cm}$ in 7 days at $25^{\circ} \mathrm{C}$.

Material examined - CHINA. Beijing, on leaf spots of Prunus avium L. (Rosaceae), 28 September 2017, K.W.T. Chethana (MFLU 18-2665, holotype) - ex-type culture, MFLUCC 181589; ibid. (KUMCC 18-0397, isotype).

Notes - Based on our phylogenetic analyses of combined ITS, GAPDH, RPB 2 , TEF 1- $\alpha$ and Alt-a sequence data of Alternaria species (Fig. 2), our strain (MFLUCC 18-1589) clustered in a clade together with the isolates of Alternaria tomato (CBS 103.30 and CBS 114.35), A. burnsii (CBS 108.27 and CBS 107.38) and A. jacinthicola (CBS 133751 and CPC 25267). Our novel taxon was separated from these taxa with high bootstrap values and strong baysean posterior probabilities (100\% MP, 95\% ML, and $1.00 \mathrm{PP}$ ). A comparison of the protein coding regions GADPH, RPB2 and TEF $1-\alpha$ between our species (MFLUCC 18-1589) and closely associated A. jacinthicola (CPC 25267) revealed $3.92 \%, 4.5 \%$ and $5.4 \%$ base pair differences respectively. Morphological comparison between them revealed different conidial characters. Compared to our strain, $A$. jacinthicola have larger conidiophores $(70 \times 2-4 \mu \mathrm{m})$ and larger $(28-32 \times 12-15 \mu \mathrm{m})$, very short beaked, 3-7 transverse and 1-2 longitudinal septate conidia (Dagno et al. 2011). As mentioned in the etymology section, our strain show a high resemblance to A. eichhorniae. When comparing our strain with the type specimen of A. eichhorniae (CBS 489.92), they are similar in morphology except for conidia and conidiomata. Our strain have slightly smaller conidia, and smaller conidiophores as compared to the type strain (Nag Raj \& Ponnappa 1970).

Glomerellaceae Locq. Wx Seifert \& W. Gams, Zhang et al., Mycologia 98(6): 1083 (2007)

Colletotrichum Corda, Sturm, Deutschl. Fl., 3 Abt. (Pilze Deutschl.) 3(12): 41. (1831)

Colletotrichum was introduced by Corda (1831) for Colletotrichum lineola Corda. The genus includes endophytes, saprobes and many plant pathogens (Hyde et al. 2009, Jayawardena et al. 2016). Colletotrichum was placed in Glomerellaceae, Sordariomycetes by Kirk et al. (2001) and this was confirmed by other studies (Maharachchikumbura et al. 2015, 2016).

Colletotrichum aenigma B.S. Weir \& P.R. Johnst., in Weir, Johnston \& Damm, Stud. Mycol. 73: 135 (2012)

Fig. 10

Facesoffungi number: FoF04914

Pathogenic on diseased leaves of Prunus avium. Sexual morph: Undetermined. Asexual morph: Pycnidia on the PDA $0.66-5 \mathrm{~mm}$ diam. $(\overline{\mathrm{x}}=2.3 \mathrm{~mm}, \mathrm{n}=10)$, black, aggregated, verrucose, sometimes reduced to hyaline conidial masses. Vegetative hyphae hyaline, smooth-walled, septate, branched. Conidiophores not observed. Conidiogenous cells poorly differentiated, arise from hyphae without any organization. Conidia $14-31.2 \times 4-8 \mu \mathrm{m}(\overline{\mathrm{x}}=18.9 \times 6.2 \mu \mathrm{m}, \mathrm{n}=40)$, hyaline, smooth-walled, aseptate, guttulate, straight, cylindrical with broadly rounded ends. Appressoria 6 $10 \mu \mathrm{m}$ diam., dark brown or black, sub-globose or with few broad lobes. Chlamydospores and setae not observed.

Culture characteristics - Colonies on PDA slow growing, attaining a diameter of $5.0 \mathrm{~cm}$ in 4 days at $25{ }^{\circ} \mathrm{C}$, circular, with cotton, dense, white aerial mycelium, reverse centre pale olivaceous grey and olivaceous grey towards the margin, becoming black with age.

Material examined - CHINA. Beijing, on leaf spots of Prunus avium L. (Rosaceae), 28 September 2017, K.W.T. Chethana (MFLU 18-2658) - living culture, MFLUCC 18-1603, KUMCC 18-0411; CHINA. Beijing, on leaf spots of Prunus avium L. (Rosaceae), 28 September 2017, K.W.T. Chethana (MFLU 18-2657) - living culture, MFLUCC 18-1604, KUMCC 18-0412.

Notes - Colletotrichum aenigma has been reported from causing diseases on a variety of hosts including Capsicum sp., Citrus sinensis (L.) Osbeck, Fragaria x ananassa Duchesne, Malus domestica Borkh., Olea europaea L., Persea americana Mill., Pyrus sp., Sedum kamtschaticum Fisch. \& C.A. Mey and Vitis vinifera L. from Asian and European regions (Schena et al. 2014, Yan 
et al. 2015, Han et al. 2016, Choi et al. 2017, Diao et al. 2017). Based on our phylogenetic analysis of combined ITS, GAPDH, CHS, ACT and TUB2 sequence data of Colletotrichum species (Fig. 3), our strain (MFLUCC 18-1603) clustered together with the ex-type strain of $C$. aenigma (ICMP 18608) with high bootstrap and Bayesian probabilities (100\% MP, 100\% ML and 1.00 PP). Comparisons of base pair differences for all the genes between our strain (MFLUCC 18-1603) and ex-type strain of $C$. aenigma (ICMP 18608) reveal identical or less than $1 \%$ base pair differences. When comparing our strain with the type specimen of C. aenigma (ICMP 18608), it showed similar morphology (Weir et al. 2012).

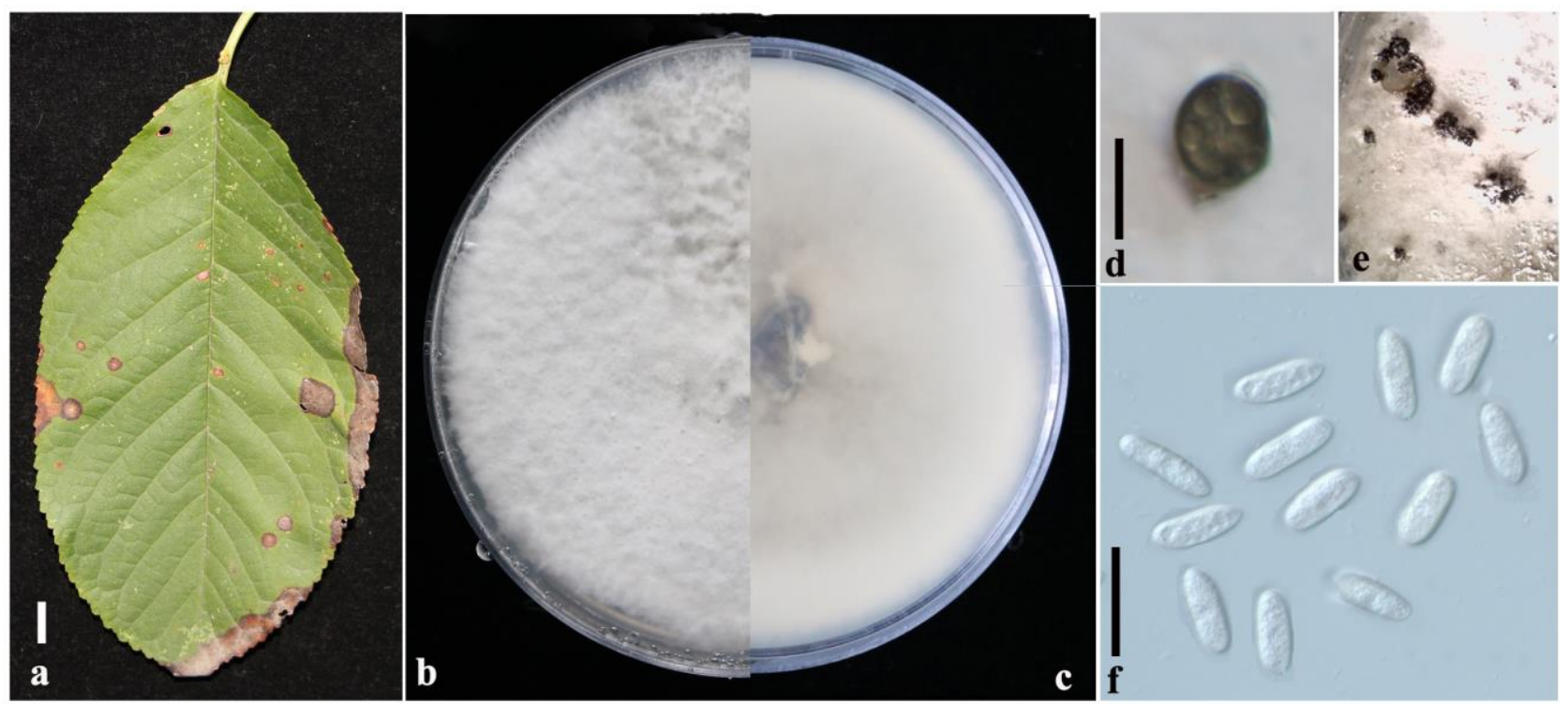

Figure 10 - Colletotrichum aenigma (MFLUCC 18-1603) a Host surface from which the pathogen was isolated. b, c Upper-view (b) and the reverse view (c) of the colony on PDA. d Appressoria. e Pycnidia on the medium. $\mathrm{f}$ Conidia. Scale bars: $\mathrm{a}=1 \mathrm{~cm}, \mathrm{~d}=10 \mu \mathrm{m}, \mathrm{f}=20 \mu \mathrm{m}$.

Colletotrichum pseudotheobromicola Chethana, Yan, Li \& K.D. Hyde, sp. nov.

Fig. 11

MycoBank number: MB828517; Facesoffungi number: FoF04915

Etymology - The specific epithet pseudotheobromicola was given after its resemblance to Colletotrichum theobromicola.

Pathogenic on diseased leaves of Prunus avium. Sexual morph: Undetermined. Asexual morph: Pycnidia on the PDA $0.4-0.97 \mathrm{~mm}$ diam. $(\overline{\mathrm{x}}=0.6 \mathrm{~mm}, \mathrm{n}=10)$, solitary, submerged in PDA, globose, black, but mostly rudimentary, reduced to orange conidial masses, forming abundantly. Vegetative hyphae hyaline to light brown, smooth-walled, septate, branched. Conidiogenous cells 8.6-14.5 $\times 1-4 \mu \mathrm{m}(\overline{\mathrm{x}}=10.9 \times 3.4 \mu \mathrm{m}, \mathrm{n}=20, \mathrm{n}=20)$, hyaline to pale brown, cylindrical, tapering uniformly from base to tip, arising from highly septate, swollen hyphae on PDA. Phialides 14.5-20 $\times 3-4 \mu \mathrm{m}$ produced from short-cell hyphae, cylindrical, tapered toward the tip and tips marked by periclinal thickening. Conidia $13-19.7 \times 4-6 \mu \mathrm{m}(\overline{\mathrm{x}}=16.6 \times 5.0 \mu \mathrm{m}, \mathrm{n}=$ 40), L/W ratio 3.3, hyaline, smooth-walled, aseptate, straight, sub-cylindrical to clavate, often with broadly rounded ends. Appressoria $6-10 \times 5-8 \mu \mathrm{m}(\overline{\mathrm{x}}=9.8 \times 6.9 \mu \mathrm{m}, \mathrm{n}=10)$, irregular, light brown. Chlamydospores and setae not observed.

Culture characteristics - Colonies on PDA slow growing, attaining a diameter of $5.0 \mathrm{~cm}$ in 4 days at $25{ }^{\circ} \mathrm{C}$, circular, with velvety to cotton, dense, greyish aerial mycelium, initially light grey, with hyaline immersed hyphae, forming dark, grey, concentric rings, becoming black with age.

Material examined - CHINA. Beijing, on leaf spots of Prunus avium L. (Rosaceae), 28 September 2017, K.W.T. Chethana (MFLU 18-2656, holotype) - ex-type culture, MFLUCC 181602; ibid. (KUMCC 18-0410, isotype). 
Notes - Based on the phylogenetic analysis of the current study of combined ITS, GAPDH, CHS, ACT and TUB2 sequence data of Colletotrichum species (Fig. 3), our taxon $C$. pseudotheobromicola (MFLUCC 18-1602) is phylogenetically distant from the ex-type strain of $C$. theobromicola (CBS 124945; 86\% MP 90\% ML and $0.98 \mathrm{PP}$ ). A comparison of the 521 nucleotides across the ITS $(+5.8 \mathrm{~S})$ gene region between $C$. pseudotheobromicola (MFLUCC 181602 ) and $C$. theobromicola (CBS 124945) reveal 3.15\% base pair difference. Furthermore, comparison of our new taxon with $C$. theobromicola (CBS 124945) for base pair differences in the protein coding genes showed, $4.74 \%$ base pair difference across 250 nucleotides in GAPDH gene region; $10 \%$ base pair difference across 282 nucleotides in CHS gene region; $3.46 \%$ base pair difference across 491 nucleotides in TUB2 gene region; and 10\% base pair difference across 256 nucleotides in ACT gene region. When comparing our strain with the type specimen of $C$. theobromicola (CBS 489.92), they are similar in morphology (Rojas et al. 2010) except for spore and appressoria characters. Our taxon C. pseudotheobromicola (MFLUCC 18-1602) differs from C. theobromicola (CBS 489.92) in having larger spores $(13-19.7 \times 4-6 \mu \mathrm{m})$ and larger appressoria $(6-10 \times 5-8 \mu \mathrm{m})$ compared to smaller spores $(14-18.7 \times 4-5 \mu \mathrm{m})$ and smaller appressoria $(6-10 \times$ 5-6 $\mu \mathrm{m})$ of $C$. theobromicola (CBS 489.92).
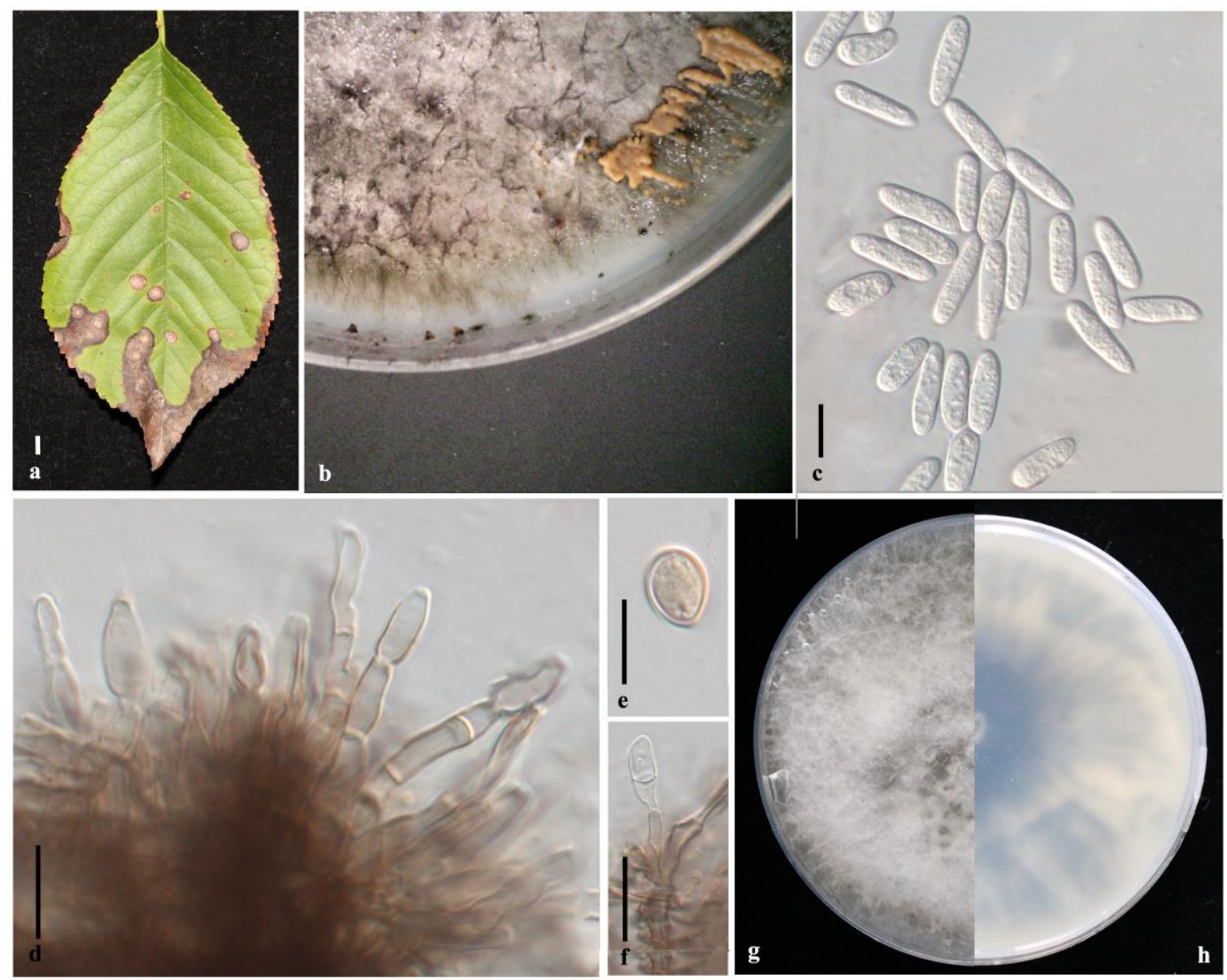

Figure 11 - Colletotrichum pseudotheobromicola (MFLUCC 18-1602, holotype) a Host surface from which the pathogen was isolated. b Pycnidia and orange colour spore mass on the PDA. c Conidia. d, f Phialides developed from septate swollen hyphae. e Appressoria. g, h Upper-view $(\mathrm{g})$ and the reverse view $(\mathrm{h})$ of the colony on PDA. $\mathrm{d}$ Conidia. Scale bars: $\mathrm{a}=7 \mathrm{~mm}, \mathrm{c}-\mathrm{f}=20 \mu \mathrm{m}$.

Didymellaceae Gruyter, Aveskamp \& Verkley, Mycol. Res. 113(4): 516 (2009)

Nothophoma Qian Chen \& L. Cai, Stud. Mycol. 82: 212 (2015)

Nothophoma was introduced by Chen et al. (2015) with Nothophoma infossa (Ellis \& Everh.)

Q. Chen \& L. Cai. to accommodate Nothophoma anigozanthi (Tassi) Q. Chen \& L. Cai., 
Nothophoma arachidis-hypogaeae (V.G. Rao) Q. Chen \& L. Cai., Nothophoma quercina (Syd.) Q. Chen \& L. Cai. and Nothophoma gossypiicola (Gruyter) Q. Chen \& L. Cai. This ubiquitous, species-rich genus includes many important plant pathogens (Chen et al. 2015).

Nothophoma pruni Chethana, Yan, Li \& K.D. Hyde, sp. nov.

Fig. 12

MycoBank number: MB828518; Facesoffungi number: FoF04917

Etymology - The specific epithet pruni was given after the host genus.

Saprobic on diseased leaves of Prunus avium. Sexual morph: Undetermined. Asexual morph: Pycnidia on the PDA surface, $0.22-0.43 \mathrm{~mm}(\overline{\mathrm{x}}=0.28 \mathrm{~mm}, \mathrm{n}=10)$ diam., solitary, scattered, globose to irregularly shaped, black, ostiolate. Conidiogenous cells phialidic, hyaline, simple, doliiform to ampulliform, variable in size. Conidia 4.8-8.5 $\times 2.7-3.9 \mu \mathrm{m}(\overline{\mathrm{x}}=6 \times 3.3 \mu \mathrm{m}, \mathrm{n}=40)$, cylindrical to obovoid or oblong, hyaline, aseptate, smooth-walled. Conidial exudates hyaline to buff.

Culture characteristics - Colonies on PDA reach $80 \mathrm{~mm}$ diam. after 7 days at $25{ }^{\circ} \mathrm{C}$, with regular margin, dull white aerial mycelium surface floccose, with reverse pale vinaceous.

Material examined - CHINA. Beijing, on leaf spots of Prunus avium L. (Rosaceae), 28 September 2017, K.W.T. Chethana (MFLU 18-2668, holotype) - ex-type culture, MFLUCC 181601; ibid. (KUMCC 18-0409, isotype); CHINA. Beijing, on leaf spots of Prunus avium L. (Rosaceae), 28 September 2017, K.W.T. Chethana (MFLU 18-2667) - living culture, MFLUCC 18-1600, KUMCC 18-0408.
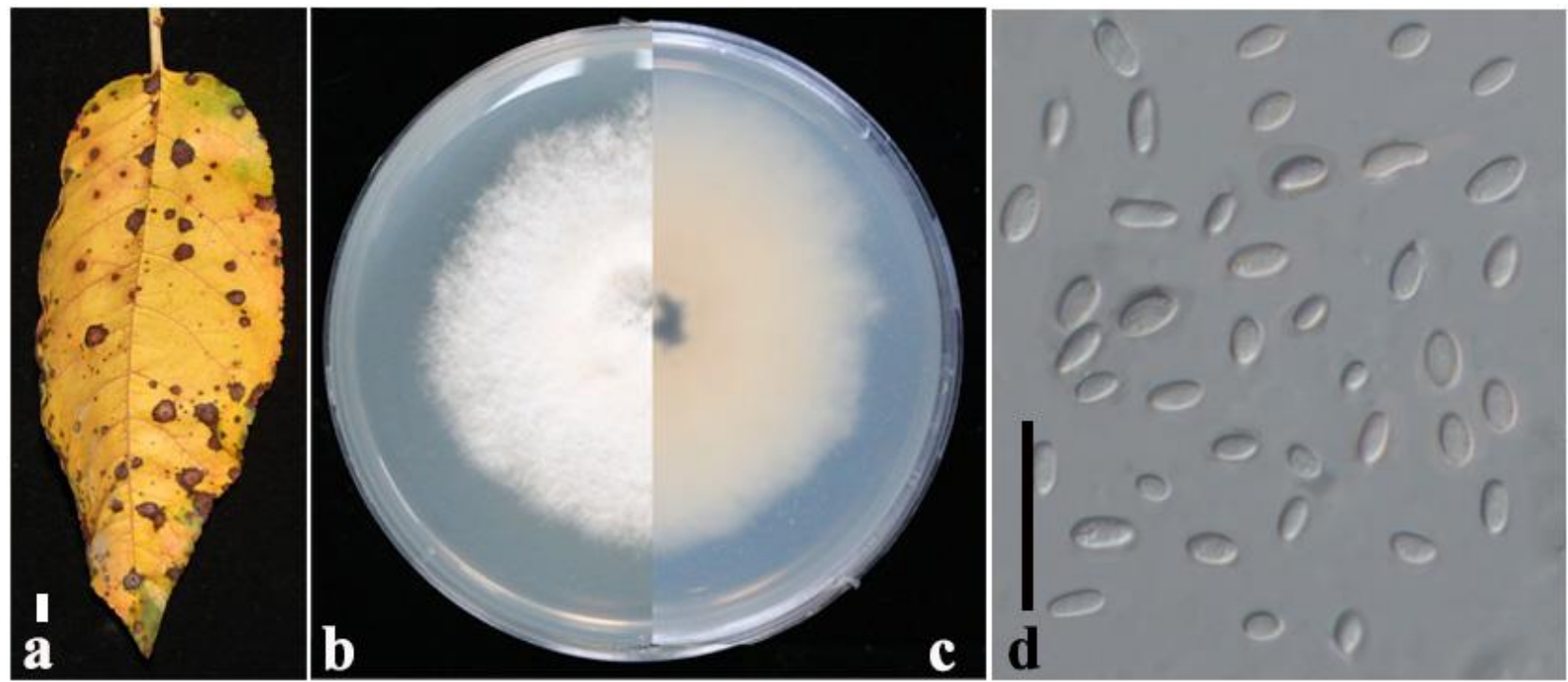

Figure 12 - Nothophoma pruni (MFLUCC 18-1601, holotype) a Host surface from which the pathogen was isolated. b, c Upper-view (b) and the reverse view (c) of the colony on PDA. d Conidia. Scale bars: $\mathrm{a}=1 \mathrm{~cm}, \mathrm{~d}=20 \mu \mathrm{m}$.

Note - According to the phylogenetic analysis of combined LSU, ITS, RPB 2 and TUB2 sequence data of Didymellaceae species (Fig. 4), our collection of Nothophoma pruni formed a subclade within genus Nothophoma. The Nothophoma pruni collection was well-separated from its sister taxa, $N$. quercina (CBS 633.92) with a relatively high bootstrap and Bayesian probabilities (95\% ML, 93\% MP, $1.00 \mathrm{PP})$. A comparison of the 486 nucleotides across the ITS (+5.8S) gene region between $N$. pruni (MFLUCC 18-1601) and $N$. quercina (CBS 633.92) reveal $2.46 \%$ base pair difference. Furthermore, comparison of our new taxon with N. quercina (CBS 633.92) for base pair differences in the protein coding genes confirmed its novelty. There are $3.19 \%$ base pair difference across 909 nucleotides in $\mathrm{RPB}_{2}$ gene region and $4.17 \%$ base pair difference across 335 nucleotides in TUB2 gene region. Our collection differs from ex-type of $N$. quercina in having cylindrical to obovoid or oblong, hyaline, slightly smaller $(4.8-8.5 \times 2.7-3.9 \mu \mathrm{m})$ conidia in 
contrast to subglobose to oval to obtuse, brown, larger $(5.5-7.5 \times 3-4.5 \mu \mathrm{m})$ conidia of $N$. quercina (Aveskamp et al. 2010).

Nothophoma quercina (Syd. \& P. Syd.) Qian Chen \& L. Cai, Stud. Mycol. 82: 213 (2015) Fig. 13 Facesoffungi number: FoF04918

Basionym: Cicinobolus quercinus Syd., Ann. Mycol. 13: 42 (1915)

$\equiv$ Ampelomyces quercinus (Syd.) Rudakov, Mikol. Fitopatol. 13: 109 (1979)

इPhoma fungicola Aveskamp et al., Stud. Mycol. 65: 26 (2010)

Saprobic on diseased leaves of Prunus avium. Sexual morph: Undetermined. Asexual morph: Pycnidia on the PDA surface, $0.22-0.43 \mathrm{~mm}(\overline{\mathrm{x}}=0.28 \mathrm{~mm}, \mathrm{n}=10)$ diam., solitary, scattered, globose to irregularly shaped, black, ostiolate. Pycnidial wall multi-layered, composed of pale brown, pseudoparenchymatous cells, thicker outer layer and thinner inner layer. Conidiogenous cells phialidic, hyaline, simple, doliiform to ampulliform, variable in size. Conidia $2-5.5 \times 1-4 \mu \mathrm{m}$ $(\overline{\mathrm{x}}=3.5 \times 2.5 \mu \mathrm{m}, \mathrm{n}=40)$, variable in size and shape, subglobose to oval or obtuse, initially hyaline, but brown at maturity, aseptate, smooth-walled. Conidial exudates hyaline to buff.
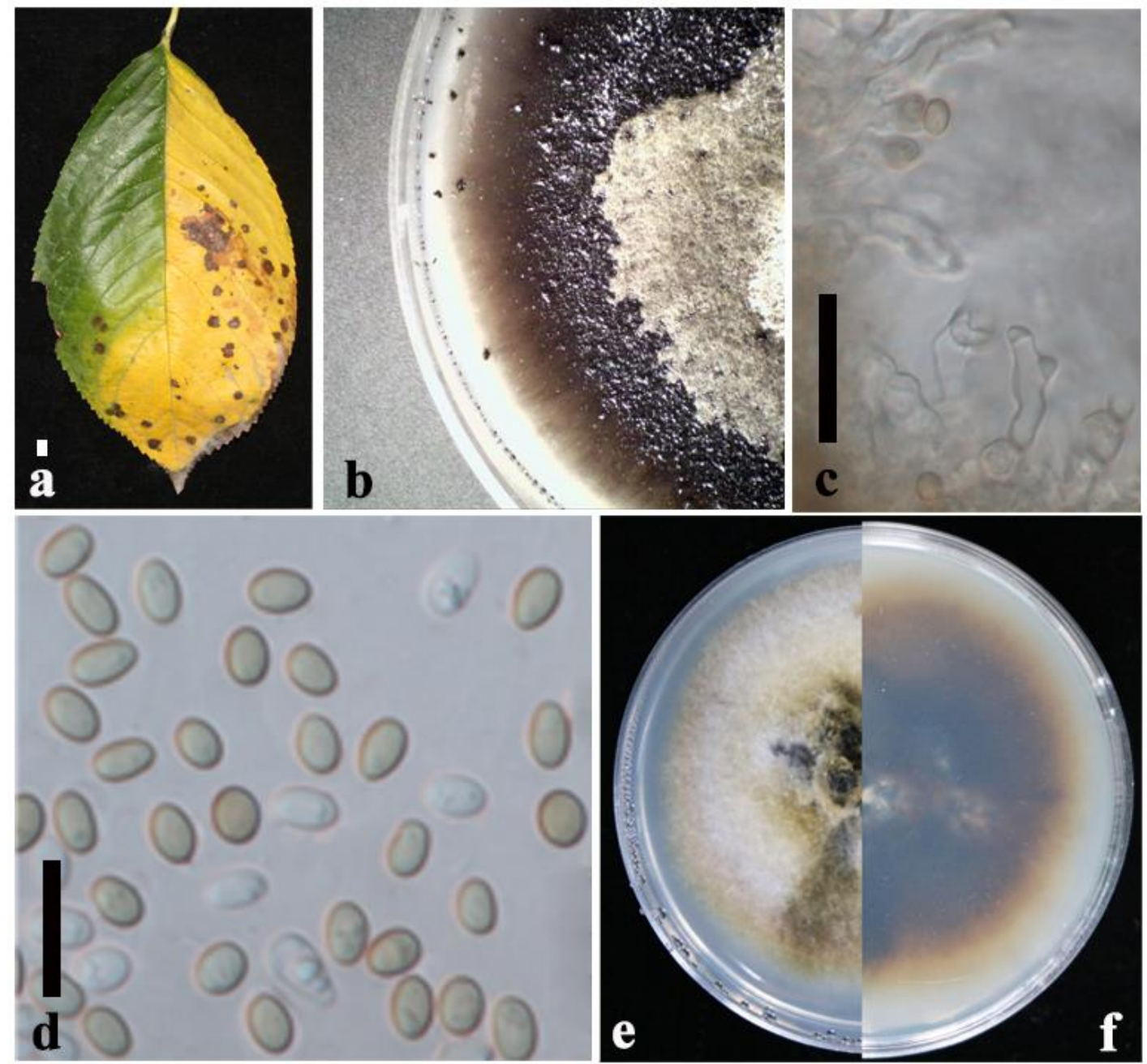

Figure 13 - Nothophoma quercina (MFLUCC 18-1588) a Host surface from which the pathogen was isolated. b Pycnidia on agar. c Conidiogenous cells. d Conidia. e, f Upper-view (e) and the reverse view (f) of the colony on PDA. Scale bars: $a=3 \mathrm{~mm}, \mathrm{c}, \mathrm{d}=20 \mu \mathrm{m}$.

Culture characteristics - Colonies on PDA reach $80 \mathrm{~mm}$ diam. after 7 days at $25{ }^{\circ} \mathrm{C}$, with regular margin, dull white aerial mycelium surface floccose to wooly, with greenish olivaceous to olivaceous near the centre and reverse dark ochreous in the centre and white in the margin. 
Material examined - CHINA. Beijing, on leaf spots of Prunus avium L. (Rosaceae), 28 September 2017, K.W.T. Chethana (MFLU 18-2666) - living culture, MFLUCC 18-1588, KUMCC 18-0396.

Note - Nothophoma quercina has been reported as a saprobe from Erysiphe alphitoides Griffon \& Maubl., Quercus sp., Ulmus sp. and Ziziphus jujuba Mill. (Aveskamp et al. 2010, Chen et al. 2015, Jianyu et al. 2016) and as a pathogen from Chaenomeles sinensis (Thouin) Koehne, Malus micromalus Makino, Phellodendron amurense Rupr. and Pistacia vera L. (Yun et al. 2016, Jiao et al. 2017, Liu et al. 2018, Moral et al. 2018). This is the first record of $N$. quercina reported on Prunus avium. When comparing our species with the type specimen of $N$. quercina (CBS 633.92), they are similar in morphology but differ in their host. The conidiomata are larger in size while conidia are smaller in size compared to the type specimen. Based on our phylogenetic analysis of combined ITS, LSU, TUB2 and RPB2 sequence data of Didymellaceae species (Fig. 4), our strain (MFLUCC 18-1588) clustered together with the ex-type strain of $N$. quercina (CBS 633.92) with relatively high bootstrap and Bayesian probabilities (100\% ML, 100\% MP, $1.00 \mathrm{PP}$ ). Base pair comparisons for all the genes between our strain (MFLUCC 18-1588) and ex-type strain of $N$. quercina (CBS 633.92) reveal identical or less than $1 \%$ base pair differences.

Stagonosporopsis Died., Annls. Mycol. 10(2): 142 (1912)

Stagonosporopsis Died. was introduced by Diedicke (1912) to separate taxa which occasionally form multi-septate taxa from Ascochyta. As no type material was specified, $S$. actaeae, the first species described by Diedicke was considered as the generic type (Boerema et al. 1997, 2004). Aveskamp et al. (2010) recombined Stagonosporopsis synanamorphs and proposed an emended description for Stagonosporopsis.

Stagonosporopsis citrulli M.T. Brewer \& J.E. Stewart, in Stewart, Turner \& Brewer, Fungal Biol. 119: 377 (2015)

Fig. 14

Facesoffungi number: FoF 04919

Saprobic on diseased leaves of Prunus avium. Sexual morph: Undetermined. Asexual morph: Pycnidia on the PDA surface, solitary, globose to subglobose, black, immersed or on the surface. Pycnidial wall multi-layered, composed of pale brown, pseudoparenchymatous cells, thicker outer layer, and a thinner inner layer. Conidiogenous cells phialidic, hyaline, simple, doliiform to ampulliform, variable in size. Conidia 4-7.6 $\times 1.6-3.5 \mu \mathrm{m}(\overline{\mathrm{x}}=6.0 \times 2.5 \mu \mathrm{m}, \mathrm{n}=40)$, cylindrical to ellipsoidal, hyaline, aseptate, straight to slightly curved, thin and smooth-walled. Conidial exudates buff.

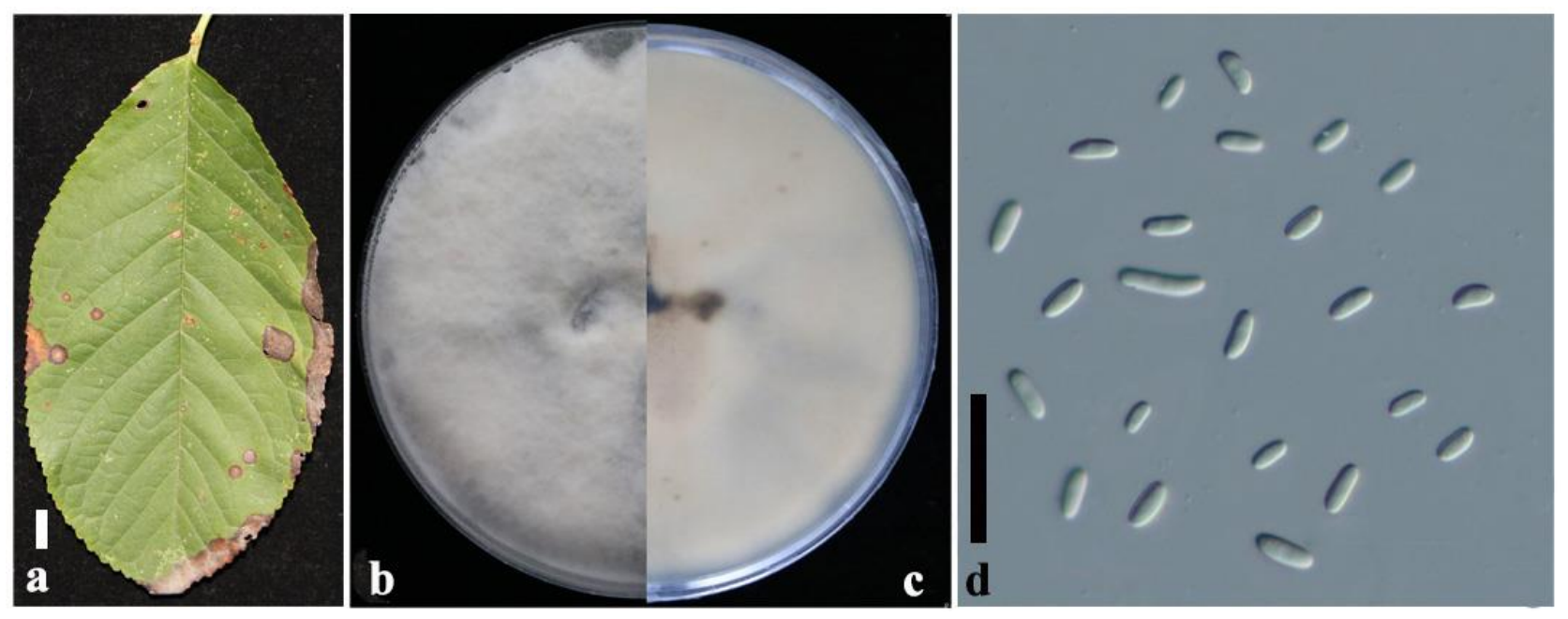

Figure 14 - Stagonosporopsis citrulli (MFLUCC 18-1595) a Host surface from which the pathogen was isolated. b, c Upper-view (b) and the reverse view (c) of the colony on PDA. d Conidia. Scale bars: $a=1 \mathrm{~cm}, \mathrm{~d}=20 \mu \mathrm{m}$. 
Culture characteristics - Colonies on PDA reach $60 \mathrm{~mm}$ diam. after 7 days at $25{ }^{\circ} \mathrm{C}$, with regular margin, floccose, white aerial mycelium, with pale olivaceous grey reverse.

Material examined - CHINA, Beijing, on leaf spots of Prunus avium L. (Rosaceae), 28 September 2017, K.W.T. Chethana (MFLU 18-2669) - living culture, MFLUCC 18-1595, KUMCC 18-0403.

Note - In the current study, a new host record was identified for S. citrulli by the phylogenetic analysis of combined ITS, LSU, TUB2 and RPB2 sequence data of Didymellaceae species (Fig. 4). Stagonosporopsis citrulli (MFLUCC 18-1595) clusters together with the ex-type of $S$. citrulli (ATCC TSD-2) with relatively high bootstrap and Bayesian probabilities (100\% ML, $100 \%$ MP, $1.00 \mathrm{PP})$. A comparison of the 499 nucleotides across the ITS $(+5.8 \mathrm{~S})$ gene region between S. citrulli (JZB380014) and S. citrulli (ATCC TSD-2) reveal 1.4\% base pair difference. Furthermore, comparison of our new tax on with S. citrulli (ATCC TSD-2) for base pair differences in the protein coding genes confirmed that it is the same species. There are no base pair difference across 324 nucleotides in TUB2 gene region. When we compared the morphology of our isolate with S. citrulli (ATCC TSD-2), both exhibited similar morphological characters (Boerema et al. 2004).

Epicoccum Link, Mag. Gesell. Naturf. Freunde, Belin 7: 32 (1816)

The genus Epicoccum was emended by Chen et al. (2015) to incorporate several Phoma species with epicoccoid, subcylindrical conidia, and irregular pycnidial conidiomata.

Epicoccum pseudokeratinophilum Chethana, Yan, Li \& K.D. Hyde, sp. nov.

Fig. 15

MycoBank number: MB828519; Facesoffungi number: FoF04916

Etymology - The specific epithet pseudokeratinophilum was given after its resemblance to Epicoccum keratinophilum.

Saprobic on diseased leaves of Prunus avium. Sexual morph: Undetermined. Asexual morph: Pycnidia on the PDA surface, $1.3-2.7 \mathrm{~mm}(\overline{\mathrm{x}}=1.8 \mathrm{~mm}, \mathrm{n}=10)$ diam., solitary, aggregated, glabrous, subglobose, brown, superficial or immersed in the media. Pycnidial wall multi-layered, composed of brown to dark brown cells of textura angularis. Conidiogenous cells phialidic, hyaline, simple, ampulliform to globose. Conidia 4.8-6.9 $\times 1.3-3 \mu \mathrm{m}(\overline{\mathrm{x}}=5.9 \times 2.2 \mu \mathrm{m}, \mathrm{n}=40)$, cylindrical to ellipsoidal, hyaline, aseptate, straight to slightly curved, thin and smooth-walled, mostly with 2 polar guttules and sometimes 3-guttules. Chlamydospores 3.5-5.2 $\times 2.9-3.6 \mu \mathrm{m}(\overline{\mathrm{x}}=$ $4.4 \times 3.2 \mu \mathrm{m}, \mathrm{n}=10$ ), unicellular, pale brown, smooth-walled, disposed singly, globose to subglobose.

Culture characteristics - Colonies on PDA reach $60 \mathrm{~mm}$ diam. after 7 days at $25{ }^{\circ} \mathrm{C}$, with regular margin, flattened, dark grey olivaceous surface towards the margin and white in the centre, dark olivaceous black reverse with grey olivaceous margin. Hyphae pale brown, smooth- and thinwalled, septate, $2.5-5 \mu \mathrm{m}$ wide.

Material examined - CHINA. Beijing, on leaf spots of Prunus avium L. (Rosaceae), 28 September 2017, K.W.T. Chethana (MFLU 18-2670, holotype) - ex-type culture, MFLUCC 181593; ibid. (KUMCC 18-0401, isotype).

Note - In our study, we identified an Epicoccum species with morphological characters similar to Epicoccum keratinophilum. When comparing our species with the type specimen of E. keratinophilum (UTHSC: DI16-271), they are similar in morphology. However, conidia are slightly larger in size as compared to the type specimen $(4-6 \times 1.5-2 \mu \mathrm{m})$ and chlamydospores were present in our culture. Based on our phylogenetic analysis of combined ITS, LSU, TUB2 and RPB2 sequence data of Epicoccum species (Fig. 4), our strain of E. pseudokeratinophilum (MFLUCC 181593) clustered basal to the ex-type strain of Epicoccum keratinophilum (UTHSC:DI16-271) with relatively high bootstrap and Bayesian probabilities (90\% ML, $98 \% \mathrm{MP}, 0.98 \mathrm{PP}$ ). A comparison of the 486 nucleotides across the ITS $(+5.8 \mathrm{~S})$ gene region between E. pseudokeratinophilum (MFLUCC 18-1593) and Epicoccum keratinophilum (UTHSC: DI16-271) reveal $2.45 \%$ base pair difference. Furthermore, comparison of our new taxon with Epicoccum keratinophilum (UTHSC: 
DI16-271) for base pair differences in the protein coding genes confirmed its novelty. There are $2.18 \%$ base pair difference across 596 nucleotides in $\mathrm{RPB}_{2}$ gene region and $5.11 \%$ base pair difference across 327 nucleotides in TUB2 gene region.

\section{Discussion}

With varying severity in different geographical and climatic regions, cherry leaf spot thrives throughout the world with moderately wet conditions and with temperatures above $16{ }^{\circ} \mathrm{C}$ (Ogawa \& English 1991, Holb 2009, Farr \& Rossman 2011, Faust \& Surányi 2011, Joshua 2012, Choi et al. 2014). The optimal temperature range for the spread of most of the pathogens is between $16-20{ }^{\circ} \mathrm{C}$ (Wilcox 1993, Pederson et al. 2012). Cherry leaf spot is identified as a common disease in Chinese orchards. In China, the fruiting period of cherry falls within the summer rainy season, facilitating the disease spread among the Chinese orchards. During the early summer, initial symptoms appear on the upper surface of leaves and with frequent rains in May and June, fungi spread extremely quickly similar to the observations by Ellis (2008). Disease severity in the orchards differ according to different environmental conditions and sanitary conditions inside the orchards.
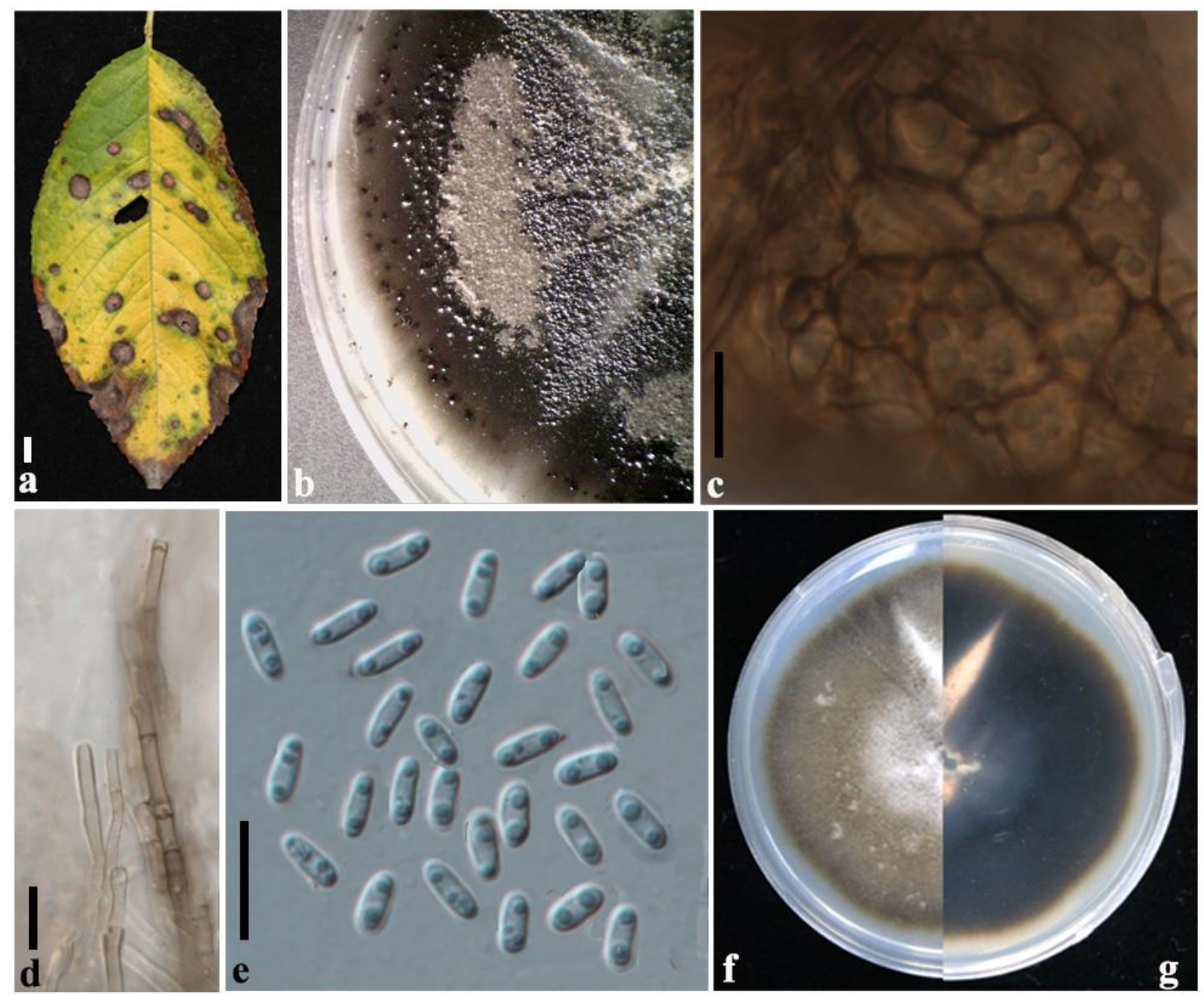

Figure 15 - Epicoccum pseudokeratinophilum (MFLUCC 18-1593, holotype) a Host surface from which the pathogen was isolated. b Pycnidia on the PDA. c Pycnidial wall. d Septate hyphae. e Conidia. f, g Upper-view (f) and the reverse view (g) of the colony on PDA. Scale bars: $a=5$ $\mathrm{mm}, \mathrm{c}-\mathrm{e}=10 \mu \mathrm{m}$.

In the current study, we have obtained 67 isolates from the diseased cherry leaves. The majority belonged to Alternaria prunicola (50 isolates) and during the pathogenicity assay, these isolates reproduced identical disease symptoms on detached leaves. Thus, we concluded $A$. 
prunicola as the causative agent of the cherry leaf spot in cherry orchards in Beijing. In addition, six isolates of A. alternata were isolated from diseased leaves. Alternaria alternata has previously been identified as one of the pathogens of Cherry leaf spot in Greece (Thomidis \& Tsipouridis 2006) and as the pathogen of black spot disease on cherry fruits (Zhao \& Liu 2012). Even though A. prunicola is the main pathogen, Colletotrichum aenigma and C. pseudotheobromicola exhibited a higher level of virulence as compared to A. prunicola in the pathogenicity assay. This can be justified as these Colletotrichum species are considered to be pathogenic on most of their hosts (Jayawardena et al. 2016). Furthermore, identification of several Didymellaceae species, which are proven to be weakly pathogenic or non-pathogenic during our pathogenicity assay, confirms that these species may cause secondary infections on diseased leaves with cherry leaf spots. In our pathogenicity assay, the disease severity of the cherry leaf spot significantly changed among the cultivars. Prunus avium cv. 'Sunburst' exhibited significantly higher resistance as compared to the other two cultivars. Therefore, cultivation of resistant cultivars such as 'Sunburst' can slightly reduce the severity of the disease in the orchards.

For most of the plant diseases, primary inoculum mostly comes from within the orchard through leaf debris, infected plant tissues and from fallen mummified berries. Similarly, for cherry leaf spot, we believe that the primary inoculum came from within the orchard. During our field survey, we observed diseased leaves among the leaf debris on the ground. Furthermore, we were able to isolate $A$. prunicola from the leaf debris, the same pathogen that we isolated from diseased leaves, confirming our observations. Thus, A. prunicola can be considered as a true pathogen. It not only relies on the host ( $P$. avium leaves) for its growth, but also capable of surviving by overwintering in the leaf debris and contribute significantly in forming the primary inoculum for the disease for the next season. Therefore, if the orchard had been infected previously by the leaf spot pathogen, there is a higher probability for the disease to occur in the new season if proper control measures are not followed. Information on the primary inoculum source, the timing of infection, proper agricultural practices as well as the application of fungicides can help the growers in designing effective disease management strategies. Since the main form of survival for the pathogen is in the leaf litter, removing and destroying the leaf litter can significantly decrease the primary inoculum. Furthermore, pruning is another method practiced by growers to reduce humidity and increase air and light circulation in the orchards. In addition to these agricultural practices, growers and researchers are experimenting the efficacy of different fungicides against cherry leaf spot pathogens (Hamilton et al. 1956, Eisensmith \& Jones 1981, Green et al. 2006).

To our knowledge, this is the first study that has identified and characterised the cherry leaf spot pathogen, A. prunicola in Beijing, China by morpho-molecular studies and pathogenicity assays. Identification of this new pathogen is important as it is the most critical step in the early detection and monitoring stages of any disease management program (Riley et al. 2002). Correct identification of the pathogen, A. prunicola, becomes important as there are many Alternaria species occurring on Cherries with varied virulence and varied lifestyles such as pathogens and saprobes (Zhu \& Chang 2004, Thomidis \& Tsipouridis 2006, Farr \& Rossman 2013, Choi et al. 2014). Identification of a new taxon indicates that a new pathogen has been evolved under the existing chemical and traditional control methods. Therefore, a new control strategy must be designed by combining chemical and agricultural approaches. Studies must be designed to confirm the efficacy of traditional fungicides such as Captan, Chlorothalonil, Tebuconazole, and Trifloxystrobin (Joshua 2012) against leaf spot pathogen A. prunicola. Furthermore, representative isolates used in our pathogenicity study (JZB3180002, MFLUCC 18-1598, MFLUCC 18-1596 and MFLUCC 18-1597, MFLUCC 18-1599) could be utilized in screening effective fungicides against our new pathogenic taxon. Another successful preventive strategy is to cultivate cherry leaf spot resistant cultivars (Riley et al. 2002). However, according to the previous research conducted against cherry leaf spot caused by Blumeriella jaapii, all the sweet cherry cultivars were susceptible to the disease, whereas several sour cherry cultivars such as 'Morina', 'Köröser Gierstädt', 'Hartai' and 'Karneol' showed some resistance (Schuster \& Tobutt 2004). In addition to integrating chemical control with proper agricultural practices, a diversified leaf spot disease management 
strategy can be implemented in cherry orchards in Beijing. In the current study, we only investigated cherry growing areas in Beijing. In future studies, the disease sample collection area should be further expanded to all the provinces in China. If possible, Alternaria cerasi cultures previously identified in China based only on morphological characters should be re-investigated. Research can now focus on species population dynamics and disease epidemiology to design more effective disease management strategies against these pathogens.

\section{Acknowledgements}

The project was funded by Beijing Talent Program for Dr. Jiye Yan and JNKYT201605. We would like to thank cherry cultivators for providing disease samples, R. S. Jayawardena and Ms. Wang Jing for their assistance in sample collection and providing isolates for the study. K.D. Hyde thanks Kunming Institute of Botany, Chinese Academy of Sciences (project 2013T2S0030, for the Visiting Professorship for Senior International Scientists.

\section{References}

Abdel-Wahab FA, Bahkali AHA, El-Gorban AM, Hodhod MS. 2017 - Natural products of Nothophoma multilocularis sp. nov. an endophyte of the medicinal plant Rhazya stricta. Mycosphere 8, 1185-1200.

Ariyawansa HA, Thambugala KM, Manamgoda DS, Jayawardena R et al. 2015 - Towards a natural classification and backbone tree for Pleosporaceae. Fungal Diversity 71, 85-139.

Aveskamp MM, de Gruyter J, Woudenberg JHC, Verkley GJM, Crous PW. 2010 - Highlights of the Didymellaceae: a polyphasic approach to characterize Phoma and related pleosporalean genera. Studies in Mycology 65, 1-60.

Berbee ML, Pirseyedi M, Hubbard S. 1999 - Cochliobolus phylogenetics and the origin of known, highly virulent pathogens, inferred from ITS and glyceraldehyde-3-phosphate dehydrogenase gene sequences. Mycologia 91, 964-977.

Boerema GH, de Gruyter J, Noordeloos ME. 1997 - Contributions towards a monograph of Phoma (Coelomycetes) - IV. Section Heterospora: Taxa with large sized conidial dimorphs, in vivo sometimes as Stagonosporopsis synanamorphs. Persoonia 16, 335-371.

Boerema GH, De Gruyter J, Noordeloos ME, Hamers MEC. 2004 - Phoma identification manual: differentiation of specific and intraspecific taxa in culture. CABI Publishing, Wallingford.

Carbone I, Kohn LM. 1999 - A method for designing primer sets for speciation studies in filamentous ascomycetes. Mycologia 91, 553-556.

Chen Q, Jiang JR, Zhang GZ, Cai L, Crous PW. 2015 - Resolving the Phoma enigma. Studies in Mycology 82, 137-217.

Chen Q, Hou LW, Duan WJ, Crous PW, Cai L. 2017 - Didymellaceae revisited. Studies in Mycology 87, 105-159.

Chethana KWT, Zhou Y, Zhang W, Liu M, Xing QK et al. 2017. Coniella vitis sp. nov. is the common pathogen of white rot in Chinese vineyards. Plant Disease 101, 2123-2136.

Choi HW, Lee YK, Hong SK. 2017 - First report of Colletotrichum aenigma causing anthracnose on Sedum kamtschaticum in Korea. Plant Disease 101, 2150.

Choi IY, Braun U, Park JH, Shin HD. 2014. First report of leaf spot caused by Pseudocercospora pruni-persicicola on Sweet Cherry in Korea. Plant Disease 98, 693.

Crous PW, Slippers B, Wingfield MJ, Rheeder J, Marasas WFO et al. 2006 - Phylogenetic lineages in the Botryosphaeriaceae. Studies in Mycology 55, 235-253.

Crous PW, Wingfield MJ, Burgess TI, Carnegie AJ, Hardy GEStJ et al. 2017 - Fungal Planet description sheets: 625-715. Persoonia 39, 270-467.

Dagno K, Crovadore J, Lefort F, Lahlali R, Lassois L et al. 2011 - Alternaria jacinthicola, a new fungal species causing blight leaf disease on water hyacinth [Eichhornia crassipes (Martius) Solms-Laubach]. Journal of Yeast Fungal Research 2, 99-105. 
Diao YZ, Zhang C, Liu F, Wang WZ, Liu L et al. 2017 - Colletotrichum species causing anthracnose disease of chili in China. Persoonia 38, 20-37.

Díaz R, Zas R, Fernández-lópez, J. 2007 - Genetic variation of Prunus avium in susceptibility to cherry leaf spot (Blumeriella jaapii) in spatially heterogeneous infected seed orchards. Annals of Forest Science 64, 21-30.

Diedicke H. 1912 - Die Abteilung Hyalodidymae der Sphaerioideen. Annals of Mycology 10, 135152.

Eisensmith SP, Jones AL. 1981 - A model for detecting infection periods of Coccomyces hiemalis on sour cherry. Phytopathology 71, 728-732.

Ellis MB. 1971 - Dematiaceous hyphomycetes. Commonwealth Mycological Institute, Kew, UK.

Ellis AM. 2008 - Cherry leaf spot. Fact Sheet Agriculture and Natural Resources. Ohio State University Extension, Columbus OH, USA. Accessed on June 2, 2018, at URL: https://ohioline.osu.edu.

Farr DF, Rossman AY. 2013 - Fungal Databases. Systematic Mycology and Microbiology Laboratory, Online publication, ARS, USDA, Accessed on August 24, 2018.

Faust M, Surányi D. 2011 - Origin and Dissemination of Cherry. In: Janick, J. 2011 - Origin and Dissemination of Prunus Crops: Peach, Cherry, Apricot, Plum, Almond. International Society for Horticultural Science, Belgium.

FAO. 2018 - FAOSTAT database collections. Food and Agriculture Organization of the United Nations. Rome. Accessed on August 24, 2018, at URL: http://faostat.fao.org.

Garcia SM, Jones AL. 1993 - Influence of temperature on apothecial development and ascospore discharge by Blumeriella jaapii. Plant Disease 77, 776-779.

Green H, Bengtsson M, Duval X, Pedersen HL, Hockenhull J et al. 2006 - Influence of urea on the cherry leaf spot pathogen, Blumeriella jaapii, and on microorganisms in decomposing cherry leaves. Soil Biology \& Biochemistry 38, 2731-2742.

Hall TA. 1999 - BioEdit: a user-friendly biological sequence alignment editor and analysis program for Windows 95/98/NT. Nucleic Acids Symposium Series 41, 95-98.

Hamilton JM, Szkolnik M, Sondheimer E. 1956 - Systemic control of cherry leaf-spot fungus by foliar sprays of Actidione derivatives. Science 123, 1175-1176.

Han YC, Zeng XG, Xiang FY, Ren L, Chen FY et al. 2016 - Distribution and characteristics of Colletotrichum spp. associated with anthracnose of strawberry in Hubei, China. Plant Disease 100, 996-1006.

Heald FD. 1993 - Manual of Plant Diseases, McGraw-Hill, New York.

Higgins BB. 1914 - Contribution to the life history and physiology of Cylindrosporium on stone fruit. American Journal of Botany 1, 145-166.

Hillis DM, Bull JJ. 1993 - An empirical test of bootstrapping as a method for assessing confidence in phylogenetic analysis. Systematic Biology 42, 182.

Holb IJ. 2009 - Some biological features of cherry leaf spot (Blumeriella jappii) with special reference to cultivar susceptibility. International Journal of Horticultural Sciences 15, 91-93.

Hong SG, Cramer RA, Lawrence CB, Pryor BM. 2005 - Alt-a 1 allergen homologs from Alternaria and related taxa: analysis of phylogenetic content and secondary structure. Fungal Genetics \& Biology 42, 119-129.

Hyde KD, Cai L, Canon PE, Crouch JA, Crous PW et al. 2009 - Colletotrichum: a catalogue of confusion. Fungal Diversity 39, 1-17.

Hyde KD, Norphanphoun C, Abreu VP, Bazzicalupo A, Chethana KWT et al. 2017 - Fungal diversity notes 603-708: Taxonomic and phylogenetic notes on genera and species. Fungal Diversity 87, 1-235.

Hyde KD, Chaiwan N, Norphanphoun C, Boonmee S, Camporesi E et al. 2018 - Mycosphere notes 169-224. Mycosphere 9, 271-430.

Index Fungorum 2018 - Accessed on August 22, 2018, at URL: http://www.indexfungorum.org/. 
Jayasiri SC, Hyde KD, Ariyawansa HA, Bhat DJ, Buyck B et al. 2015 - The Faces of Fungi database: fungal names linked with morphology, phylogeny and human impacts. Fungal Diversity 74, 3-18.

Jayasiri SC, Hyde KD, Jones EBG, Jeewon R. 2017 - Taxonomy and multigene phylogenetic evaluation of novel species in Boeremia and Epicoccum with new records of Ascochyta and Didymella (Didymellaceae). Mycosphere 8, 1080-1101.

Jayawardena RS, Hyde KD, Damm U, Cai L, Liu M et al. 2016 - Notes on currently accepted species of Colletotrichum. Mycosphere 7, 1192-1260.

Jeewon R, Hyde KD. 2016 - Establishing species boundaries and new taxa among fungi: recommendations to resolve taxonomic ambiguities. Mycosphere 7, 1669-1677.

Jianyu B, Xiaoming W, Yanjiang S, Canxing D. 2016 - Occurrence and identification of Nothophoma quercina causing brown spot of jujube in China. Canadian Journal of Plant Pathology 38, 527-532.

Jiao WL, Zhou RJ, Fu JF, Xu HJ, Hao N. 2017 - First report of Nothophoma quercina causing leaf spot disease of Phellodendron amurense in China. Plant Disease 101, 1820-1821.

Jones AL, Sutton TB. 1996 - Diseases of tree fruits in the east. Northcentral Regional Bulletin 45, Michigan State University.

Joshua J. 2012 - Studies on the Cherry Leaf Spot Disease in Flowering Cherries (Prunus species). $\mathrm{PhD}$ Thesis, Tennessee State University, Tennessee, United States.

Joshua J, Mmbaga MT. 2013 - Perpetuation of Cherry Leaf Spot Disease in Ornamental Cherry. Journal of Phytopathology 163, 194-201.

Katoh K, Toh H. 2008 - Recent developments in the MAFFT multiple sequence alignment program. Briefings in Bioinformatics 9, 276-285.

Khan NA, Bhat MA, Ahmad K, Qazi NA. 2014 - First record of Cherry Leaf Spot caused By Cylindrosporium padi (P. Karst.) from India. Applied Biological Research 16, 122-123.

Kirk PM, Cannon PF, David JC, Stalpers JA. 2001 - Ainsworth and Bisby's dictionary of the fungi, 8th edn. CABI Publishing, London.

Kishino H, Hasegawa M. 1989 - Evaluation of the maximum likelihood estimate of the evolutionary tree topologies from DNA sequence data. Journal of Molecular Evolution 29, 170-179.

Li M, Qi W, Cai QX, Guang DX, Yan Z et al. 2011 - Symptoms and treatment of leaf diseases of Cherry (in Chinese). Moderate Rural Science Technology 9, 35.

Liu YL, Whelen S, Hall BD. 1999 - Phylogenetic relationships among ascomycetes: evidence from an RNA polymerase II subunit. Molecular Biology \& Evolution 16, 1799-1808.

Liu M, Zhang W, Manawasinghe IS, Zhou Y, Xing QK et al. 2018 - First report of Nothophoma quercina causing trunk canker on crab apple (Malus micromalus) in China. Plant Disease 102, 1462.

Maharachchikumbura SN, Hyde KD, Jones EBG, McKenzie EHC, Huang SK et al. 2015 Towards a natural classification and backbone tree for Sordariomycetes. Fungal Diversity 72, 199-301.

Maharachchikumbura SN, Hyde KD, Jones EBG, McKenzie EHC, Jayarama B et al. 2016 Families of Sordariomycetes. Fungal Diversity 79, 1-317.

McAlpine D. 1902 - Fungus Diseases of stone-fruit trees in Australia and their treatment. Department of Agriculture, Victoria, Australia.

Moral J, Lichtemberg PSF, Papagelis A, Sherman J, Michailides TJ. 2018 - Didymella glomerata causing leaf blight on pistachio. European Journal of Plant Pathology 151, 1095-1099.

Nag Raj TR, Ponnappa KM. 1970 - Blight of water-hyacinth caused by Alternaria eichhorniae sp. nov. Transactions of the British Mycological Society 55, 123-130.

Nees von Esenbeck CG. 1816 - Das system der pilze und schwämme. Wurzburg, Germany.

Nylander JAA. 2004 - MrModeltest 2.0. Program distributed author. Evolutionary Biology Centre, Uppsala University. 
O’Donnell K, Cigelnik E. 1997 - Two divergent intragenomic rDNA ITS2 types within a monophyletic lineage of the fungus Fusarium are nonorthologous. Molecular Phylogenetics and Evolution 7, 103-116.

Ogawa JM, English H. 1991 - Diseases of temperate zone tree fruit and nut crops. Division of Agriculture and Natural Resources, University of California, Oakland, USA.

Page RDM. 1996 - TreeView: an application to display phylogenetic trees on personal computers. Computer Applications in the Biosciences 12, 357-358.

Pedersen HL, Jensen B, Munk L, Bengtsson MV, Trapman M. 2012 - Reduction in the use of fungicides in apple and sour cherry production by preventative methods and warning systems. Pesticides Research 139, 14-71.

Rehner SA, Samuels GJ. 1994 - Taxonomy and phylogeny of Gliocladium analysed from nuclear large subunit ribosomal DNA sequences. Mycological Research 98, 625-634.

Riley MB, Williamson MR, Maloy O. 2012 - Plant disease diagnosis. The Plant Health Instructor, DOI: 10.1094/PHI-I-2002-1021-01.

Rojas EI, Rehner SA, Samuels GJ, Van Bael SA, Herre EA et al. 2010 - Colletotrichum gloeosporioides associated with Theobroma cacao and other plants in Panama: multilocus phylogenies distinguish host-associated pathogens from asymptomatic endophytes. Mycologia 102, 1318-1338.

Ronquist F, Huelsenbeck JP. 2003 - MrBayes 3: Bayesian phylogenetic inference under mixed models. Bioinformatics 19, 1572.

Schena L, Mosca S, Cacciola SO, Faedda R, Sanzani SM et al. 2014 - Species of the Colletotrichum gloeosporioides and $C$. boninense complexes associated with olive anthracnose. Plant Pathology 63, 437-446.

Schuster M, Tobutt KR. 2004 - Screening of cherries for resistance to leaf spot, Blumeriella jaapii. Acta horticulture 663, 239-244.

Scott RR, Bugang W, Zhen X, Lesny V. 2014 - Annual Report on Stone Fruit in People's Republic of China. Foreign Agricultural Service, United States Department of Agriculture. USA.

Shu gui X. 2007 - Occurrence and Control of Cherry leaf spot. Proceedings of China Meeting, 2007.

Silvestro D, Michalak I. 2010 - RaxmlGUI: a graphical front-end for RAxML. Accessed on 29 September 2015, at URL http://sourceforge.net/projects/raxmlgui/

Sivanesan A. 1990 - CMI description sheets: Set 99. Mycopathlogia 109, 41-62.

Sjulin TM, Jones AL, Andersen RL. 1989 - Expression of partial resistance to cherry leaf-spot in cultivars of sweet, sour, duke, and European ground cherry. Plant Disease 73, 56-61.

Stewart JE, Turner AN, Brewer MT. 2015 - Evolutionary history and variation in host range of three Stagonosporopsis species causing gummy stem blight of cucurbits. Fungal Biology 119, 370-382.

Sung GH, Sung JM, Hywel-Jones N, Spatafora JW. 2007 - A multi-gene phylogeny of Clavicipitaceae (Ascomycota, Fungi): Identification of localized incongruence using a combinational bootstrap approach. Molecular Phylogenetics and Evolution 44, 1204-1223.

Swofford DL. 2002 - PAUP* 4.0: phylogenetic analysis using parsimony (* and other methods). Sinauer Associates, Sunderland.

Templeton MD, Rikkerink EHA, Solon SL, Crowhurst RN. 1992 - Cloning and molecular characterization of the glyceraldehyde-3-phosphate dehydrogenase encoding gene and cDNA from the plant pathogenic fungus Glomerella cingulata. Gene 122, 225-230.

Thambugala KM, Wanasinghe DN, Phillips AJL, Camporesi E, Bulgakov T et al. 2017 Mycosphere notes 1-50: Grass (Poaceae) inhabiting Dothideomycetes. Mycosphere 8, 697796.

Thomidis T, Tsipouridis C. 2006 - First Report of Alternaria Leaf Spot on Cherry Trees in Greece. Plant Disease 90, 680. 
Tibpromma S, Hyde KD, Jeewon R, Maharachchikumbura SSN, Liu JK et al. 2017 - Fungal diversity notes 491-602: taxonomic and phylogenetic contributions to fungal taxa. Fungal Diversity 83, 1-261.

Tibpromma S, Hyde KD, Bhat JD, Mortimer PE, Xu J et al. 2018 - Identification of endophytic fungi from leaves of Pandanaceae based on their morphotypes and DNA sequence data from southern Thailand. MycoKeys 33, 25-67.

USDA 2017 - USDA Global Analysis - Fresh Peaches and Cherries: World Markets and Trade. Foreign Agricultural Service, United States Department of Agriculture. USA.

Uyemoto JK, Ogawa JM, Jaffee BA. 2018 - Common Names of Plant Diseases: Diseases of Sweet Cherry (Prunus avium L.) and Sour Cherry (P. cerasus L.). The American Phytopathological Society. USA.

Valenzuela-Lopez N, Cano-Lira JF, Guarro J, Sutton DA, Wiederhold N et al. 2018 Coelomycetous Dothideomycetes with emphasis on the families Cucurbitariaceae and Didymellaceae. Studies in Mycology 90, 1-69.

Vilgalys R, Hester M. 1990 - Rapid genetic identification and mapping of enzymatically amplified ribosomal DNA from several Cryptococcus species. Journal of Bacteriology 172, 4238-4246.

Wanasinghe DN, Phukhamsakda C, Hyde KD, Jeewon R, Lee HB. 2018 - Fungal diversity notes 709-839: taxonomic and phylogenetic contributions to fungal taxa with an emphasis on fungi on Rosaceae. Fungal Diversity 89, 1-236.

Weir BS, Johnston PR, Damm U. 2012 - The Colletotrichum gloeosporioides species complex. Studies in Mycology 73, 115-180.

White TJ, Bruns T, Lee S, Taylor JW. 1990 - Amplification and direct sequencing of fungal ribosomal RNA genes for phylogenetics. In: Innis MA, Gelfand DH, Sninsky JJ, White TJ, (Eds.) PCR protocols: A guide to methods and applications. Academic, New York, pp. 315322.

Wilcox WF. 1993 - Cherry Leaf Spot. Integrated Pest Management, Cornell University. Retrieved on February 21, 2019 from www.nysipm.cornell.edu/factsheets/treefruit/diseases/cls/cls.pdf

Woudenberg JHC, Groenewald JZ, Binder M, Crous PW. 2013 - Alternaria redefined. Studies in Mycology 75, 171-212.

Woudenberg JHC, Sedi MF, Groenewald JZ, Vries Mde, Stielow JB et al. 2015 - Alternaria section Alternaria: Species, formae speciales or pathotypes?. Studies in Mycology 82, 1-21.

Yan JY, Jayawardena MMRS, Goonasekara ID, Wang Y, Zhang W et al. 2015 - Diverse species of Colletotrichum associated with grapevine anthracnose in China. Fungal Diversity 71, 233 246.

Yun YH, Oh MH. 2016 - First report of Nothophoma quercina causing shoot canker on Chaenomeles sinensis in Korea. Plant Disease 100, 2533-2534.

Zhao YZ, Liu ZH. 2012 - First report of black spot disease caused by Alternaria alternata on cherry fruits in China. Plant Disease 96, 1580.

Zhu J, Chang Y. 2004 - Cherry black ring spot pathogen identification and biological study (in Chinese). China Fruits 3, 9-12. 